\title{
Integrin $\alpha 6 \beta 4$ recognition of a linear motif of bullous pemphigoid antigen BP230 controls its recruitment to hemidesmosomes
}

\author{
José A Manso ${ }^{1}$, María Gómez-Hernández ${ }^{1}$, Arturo Carabias ${ }^{1}$, Noelia Alonso-García ${ }^{1}$, Inés García- \\ Rubio $^{2}$, Maaike Kreft ${ }^{3}$, Arnoud Sonnenberg ${ }^{3}$, and José M de Pereda ${ }^{1,4, *}$ \\ ${ }^{1}$ Instituto de Biología Molecular y Celular del Cáncer, Consejo Superior de Investigaciones Científicas - \\ University of Salamanca, Campus Unamuno, 37007 Salamanca, Spain. \\ ${ }^{2}$ Centro Universitario de la Defensa, ctra. Huesca s/n, 50090 Zaragoza, Spain. \\ ${ }^{3}$ Netherlands Cancer Institute, Plesmanlaan 121, 1066 CX Amsterdam, The Netherlands. \\ ${ }^{4}$ Lead Contact \\ *Correspondence: pereda@usal.es
}

\section{Summary}

Mechanical stability of epithelia requires firm attachment to the basement membrane via hemidesmosomes. Dysfunction of hemidesmosomal proteins causes severe skin blistering diseases. Two plakins, plectin and BP230 (BPAG1e), link the integrin $\alpha 6 \beta 4$ to intermediate filaments in epidermal hemidesmosomes. Here, we show that a linear sequence within the isoform-specific N-terminal region of BP230 binds to the third and fourth FnIII domains of $\beta 4$. The crystal structure of the complex and mutagenesis analysis revealed that BP230 binds between the two domains of $\beta 4$. BP230 induces closing of the two FnIII domains that are locked in place by an inter-domain ionic clasp required for binding. Disruption of BP230- $\beta 4$ binding prevents recruitment of BP230 to hemidesmosomes in human keratinocytes, revealing a key role of this interaction for hemidesmosome assembly. Phosphomimetic substitutions in $\beta 4$ and BP230 destabilize the complex. Thus, our study provides insights into the architecture of hemidesmosomes and potential mechanisms of regulation.

\section{Keywords}

Cell adhesion, Epithelia, Keratinocytes, Plakins, Protein-protein interactions 


\section{Introduction}

Hemidesmosomes (HDs) are junctional complexes that mediate the firm attachment of epithelial cells to the basement membrane to maintain the integrity of epithelial tissues (Walko et al., 2015). Pseudostratified and stratified epithelia, such as the epidermis, assemble classic type I HDs. These are rivet-like structures that contain three transmembrane proteins, the integrin $\alpha 6 \beta 4$, the bullous pemphigoid antigen BP180 (also known as BPAG2 or collagen XVII), and the tetraspanin CD151; and two intracellular proteins of the plakin family, plectin and BP230 (also known as BPAG1e). $\alpha 6 \beta 4$ and BP180 bind to laminin-332 in the epidermal basement membrane (Van den Bergh et al., 2011; Wilhelmsen et al., 2006), and are connected to the keratin intermediate filaments via plectin and BP230 (Geerts et al., 1999; Niessen et al., 1997a; Rezniczek et al., 1998), thereby linking the extracellular matrix to the cytoskeleton. Simple epithelia, such as that in the intestine, assemble type II HDs that contain $\alpha 6 \beta 4$ and plectin but lack $\mathrm{BP} 180$ and BP230.

The integrin $\alpha 6 \beta 4$ is an essential component of HDs and a hub of the HD protein-interaction network. Most of the intracellular interactions of $\alpha 6 \beta 4$ are established by the $\beta 4$ subunit. The $\beta 4$ cytodomain $(\sim 1000$ residues) contains a Calx $\beta$ and four fibronectin type-III domains arranged in two pairs (FnIII-1,2 and FnIII-3,4) separated by a region named the connecting segment (CS); a C-terminal tail (C-tail) extends downstream of the FnIII-4 (Figure 1A). The FnIII-1,2 and the beginning of the CS bind to the actinbinding domain (ABD) of plectin (de Pereda et al., 2009; Geerts et al., 1999). The final part of the CS and the C-tail make a second site of contact with the plakin domain of plectin (Koster et al., 2004; Rezniczek et al., 1998). The FnIII-3,4 and part of the CS bind to BP230 (Hopkinson and Jones, 2000; Koster et al., 2003). The FnIII-3 also interacts with the cytoplasmic domain of BP180 (Koster et al., 2003). The region FnIII-3,4-C-tail also interacts with Solo (ARHGEF40), a guanine nucleotide exchange factor of the RhoA small GTPase, for the formation of HDs in mammary epithelial cells (Fujiwara et al., 2018). Recently, binding of the FnIII-3 domain to L-periaxin, a myelin protein of Schwann cells, has been described (Raasakka et al., 2019).

BP230 is the epidermal-specific splice variant coded by the BPAG1/dystonin gene DST (Leung et al., 2001). BP230 has the characteristic three-segment structure shared by classic plakins. The N-terminal region contains a short isoform-specific N-terminal tail (N-tail) followed by a plakin domain $(\sim 1000$ residues) that consists of eight spectrin repeats (SR2 to SR9) and an SH3 domain (Jefferson et al., 2007; Sonnenberg et al., 2007) (Figure 1A). This region interacts with $\alpha 6 \beta 4$ and with BP180 (Koster et al., 2003).

Recruitment of BP230 into HDs requires the association of plectin with $\alpha 6 \beta 4$ and the presence of BP180 (Koster et al., 2003), suggesting that BP230 is incorporated in the final stages of HD assembly (Wilhelmsen et al., 2006). Genetic mutations in the DST gene that target BP230 cause a mild form of 
epidermolysis bullosa simplex, a blistering disease characterized by skin fragility (Groves et al., 2010). Ablation of BP230 in mice resulted in a similar phenotype of blistering caused by mechanical stress (Guo et al., 1995). In the absence of BP230, HDs lack an intracellular substructure called the inner plaque, and the bundles of keratin filaments do not attach to the HDs. The role of BP230 in the attachment of intermediate filaments is further supported by the absence of the inner plaque in type II HDs and the less robust connection of intermediate filaments to type II than to type I HDs (Uematsu et al., 1994).

HDs are dynamic complexes that disassemble when epithelial cells migrate, for example during wound healing (Gipson et al., 1993) and in invasive carcinoma cells (Herold-Mende et al., 2001). In keratinocytes, epidermal growth factor and phorbol myristate acetate promote HD disassembly by inducing phosphorylation of the $\beta 4$ subunit through the activation of the Ras/ERK-1/2 and protein kinase C (PKC) pathway (Frijns et al., 2010; Margadant et al., 2008; Rabinovitz et al., 1999). Phosphorylation of Ser residues in the CS (Frijns et al., 2010; Rabinovitz et al., 2004; Wilhelmsen et al., 2007) and in the Ctail (Frijns et al., 2012) of $\beta 4$ disrupts the interaction with plectin and is a major mechanism for HD disassembly. Phosphorylation of $\beta 4$ at S1424 correlates with a loss of co-localization with BP230 and BP180 (Germain et al., 2009); yet, little is known about the mechanisms that regulate the interaction of $\alpha 6 \beta 4$ with BP proteins during HD disassembly.

The structural understanding of protein-protein interactions in HDs is limited to the primary contact between $\alpha 6 \beta 4$ and plectin (de Pereda et al., 2009; Song et al., 2015). The isolated region FnIII-3,4 of $\beta 4$ is reluctant to crystallize, thus, we had solved its structure using hybrid methods (Alonso-Garcia et al., 2015). Nonetheless, how the FnIII-3,4 engages with BP230 or other proteins remained unknown. Here, we present a detailed mapping of the mutual binding sites in $\beta 4$ and BP230, the 3D structure of the $\beta 4-B P 230$ complex, and the conformational changes that binding causes in $\beta 4$. Finally, we have identified potentially phosphorylatable residues in BP230 and $\beta 4$ that play key roles in their interaction.

\section{RESULTS}

\section{Integrin $\beta 4$ binds to a segment of the N-terminal tail of BP230.}

Residues 1-56 of BP230 interact with the FnIII-3,4 of $\beta 4$ in yeast two-hybrid assays; and the longer segment 1-92 associated more efficiently with $\beta 4$ (Koster et al., 2003). The region 1-92 includes the N-tail (residues 1-55) and the first $\alpha$-helix of the SR2 (BP230 does not have the SR1). To investigate the binding to $\beta 4$ in vitro, we created constructs of BP230 that include the complete SR2 (56-162) to maintain the integrity of the SR fold. The fragment 1-162 of BP230 had extremely low solubility that hampered its characterization. The longer construct of this region suitable for analysis was the 10-162. Using size exclusion chromatography (SEC), interaction was detected between $\beta 4$ 1436-1666 ( $\beta 4-C S-F n I I I-3,4)$ and 
BP230 10-162, but not with the isolated SR2 (Figure S1). Thus, direct binding of BP230 to $\beta 4$ requires the N-tail.

In order to map the regions within the N-tail of BP230 responsible for binding to $\beta 4$, the affinity of $\beta 4$-CSFnIII-3,4 for a series of BP230 N-terminal deletion mutants was determined (Figure 1B,D and Table S1). $\beta 4$ bound to BP230 constructs 10-162, 19-162, and 28-162 with similar affinity, suggesting that the segment 10-27 does not contribute to the interaction. The fragments of BP230 32-162 and 37-162 had slightly lower affinity with respect to the longer constructs. Affinity for BP230 41-162 was notably lower (5-fold larger equilibrium dissociation constant, $K d$ ). Finally, BP230 46-162 and 56-162 did not bind to $\beta 4$. In summary, residues $41-45$ of BP230 are essential for binding to $\beta 4$, and residues $28-40$ also contributed to the interaction.

Despite the fact that the SR2 was not sufficient to sustain binding to $\beta 4$, we explored if it contributes to the interaction. First, we created a chimeric protein in which the SR2 of BP230, residues 59-162, was replaced by the SR2 of plectin, residues 420-530. $\beta 4-C S-F n I I I-3,4$ bound to the BP230/plectin chimera with a similar $K d$ as to the equivalent BP230 fragment 19-162, suggesting that the SR2 is not required for binding to $\beta 4$ or that its contribution can be mimicked by the SR2 of plectin.

Next, we analyzed the binding of $\beta 4$ to three synthetic peptides of the $N$-tail (Figure 1C,D). $\beta 4$ bound to the BP230 peptide 26-55 with slightly higher affinity than to the BP230 fragment 28-162 that contains the SR2; further supporting the notion that the SR2 is dispensable for the interaction. Binding of $\beta 4$ to two shorter BP230 peptides, 26-47 and 37-55, shows a 5-fold and a 3-fold increase in the $K d$ with respect to the 26-55 peptide, respectively. Thus, the segments 26-36 and 48-55 contribute to the interaction. Taken together, our data indicate that the region 26-55 of the N-tail of BP230, which is only present in this isoform of BPAG1, is sufficient for binding to $\beta 4$.

\section{Mapping the BP230-binding site in $\beta 4$}

First, we analyzed the contribution to the binding to BP230 of the distinct modules included in the BP230interaction region previously identified using yeast two-hybrid assays (Koster et al., 2003) (Figure 2A). The fragment $\beta 4$-FnIII-3,4 (1457-1666) bound to BP230 with the same affinity as the longer $\beta 4$-CS-FnIII3,4, suggesting that the CS does not participate directly in the interaction. The individual FnIII domains did not bind to BP230. Thus, the FnIII-3,4 pair is necessary and sufficient for binding to BP230.

Next, we combined structure-based site directed mutagenesis with the fluorescent-based quantitative assay to map the BP230-binding site in $\beta 4$ (Figure 2B,C). To prevent distortions of the FnIII fold, mutations were introduced at solvent-exposed residues in the structures of the FnIII-3 and FnIII-4. Initially, we mainly created reverse-charge substitutions and, when possible, we changed two or three adjacent residues. The mutants Q1479R, E1480R, R1482E/E1484R/R1485E, E1493R, N1498R, E1501R, R1504E, 
Q1512R, E1541R/E1543R, and T1547R, which carry substitutions in the FnIII-3, showed values of the $K d$ within a 2-fold range, higher or lower, with respect to the wild type (WT) $\beta 4$ protein, and were considered to have a small effect on the interaction. On the other hand, the mutants R1463E, A1468R, A1468D, R1475E, Q1535R/E1536R, and R1540E/R1542E showed a notable reduction in the affinity for BP230. The effects of reverse-charge substitutions could be caused by either the loss of contacts mediated by the WT side-chain, or by contributions of the engineered residue. To differentiate these two mechanisms, when a mutation altered significantly the affinity for BP230, we also analyzed the change to Ala. R1463A, and R1475A reduced the affinity for BP230, indicating that these two Arg play important roles for binding. To further characterize the double mutants that affect the binding, we also analyzed the individual substitutions. The single mutants Q1535R, Q1535A, E1536R, and E1536A showed a similar affinity for BP230 as the WT $\beta 4$, suggesting that individually these two residues have a minor contribution to the binding. As for the pair R1540E/R1542E, the single mutant R1540E displayed only a minor effect on the binding, while R1542E significantly reduced the affinity for BP230. Similarly, R1542A, but not R1540A, reduced the affinity.

Similarly, we analyzed the effect of substitutions of residues in the FnIII-4. The $\beta 4$ mutants V1578R/T1580R，R1595E/R1596E，D1600R，P1616R/T1618R，R1621E/D1623R，E1628R/R1630E, T1632R/P1634R, S1637R/E1638R, and F1654R had a similar affinity for BP230 as the WT protein. Only the substitution P1576R resulted in an increase in the $K d$ larger than 2-fold compared to the WT. The double mutant I1661R/T1663R had increased affinity for BP230 ( 8-fold reduction in the $K d)$. This effect was due to the change T1663R, while the mutant I1661R had a similar affinity for BP230 as the WT $\beta 4$. In contrast to the effect of T1663R, the mutant T1663D showed reduced affinity for $\beta 4$, further supporting a role of $\mathrm{T} 1663$ in the interaction.

We mapped the effect of the mutations onto the structure of the isolated FnIII-3,4 (Figure 2D). The two FnIII domains are arranged in a slightly bent manner, forming a curved shape with concave and convex sides. The mutations were widely distributed throughout the surface. Yet, the residues whose substitutions mostly altered binding to BP230 (R1463, A1468, R1475, R1542, P1576, and T1663) clustered at the centre of the concave side, suggesting that the BP230-binding area extends around the cleft formed by the two FnIII domains, which is evolutionary conserved (Alonso-Garcia et al., 2015).

\section{Structural characterization of the $\beta 4-B P 230$ complex.}

We used x-ray crystallography to unveil the structural basis of the BP230 binding to $\beta 4$. The weak interaction between BP230 and $\beta 4$ posed a major challenge to crystallize their complex. To overcome this limitation, the structure of the $\beta 4$-BP230 complex was initially elucidated using the high-affinity mutant $\beta 4-T 1663 R$ bound to BP230 26-55; this structure was solved to 1.55 Á. Subsequently, the structure of the 
$\beta 4(\mathrm{WT})$-BP230 complex was solved to $2.05 \AA$ Á. The WT and mutant complexes crystallized in the same crystal form that contains a single copy of the complex in the asymmetric unit (Table 1 and Figure 3). The two structures were almost identical; after superimposition, the root mean square displacement (rmsd) of all main-chain atoms between the two structures was 1.03 Á. Only a segment of the inter-domain linker of $\beta 4$, residues 1557-1564, shows differences between the two structures (see below). Hereafter, the WT structure is described unless otherwise indicated.

The structures of the FnIII-3 and FnIII-4 in the complex are very similar to those observed in the crystals of the isolated domains (Figure S2). The inter-domain linker of $\beta 4$, which was only approximately modelled in the structure of the free FnIII-3,4, contributes to the organization of the two FnIII domains and to the BP230-binding interface. The final part of the linker, residues $1566-1569$, form a $\beta$-strand $\mathrm{H}$ that lies between the two FnIII domains and participates in the BP230-binding site. Residues 1562-1564, upstream strand H, contact BP230 making two main-chain H-bonds. In the $\beta 4(T 1663 R)-B P 230$ complex, the linker also contacts BP230, but it adopts a slightly different conformation (Figure S2). T1663R is unlikely to cause these differences because it does not contact the linker and does not induce local changes in BP230.

Continuous electron density was observed for residues 27-49 of BP230 in the WT structure and for residues 27-50 in the T1663R structure (Figure S3). BP230 adopts an extended hairpin-like structure that contains three $\beta$-strands ( $\beta \mathrm{A}, \beta \mathrm{B}$, and $\beta \mathrm{C}$ ) (Figure 3A). The first part of BP230 (residues 27-41) packs against one side of the FnIII-4; strands $\beta$ A (29-33) and $\beta$ B (39-40) make $\beta$-contacts with strands G2 and A of the FnIII-4, respectively (Figure 3B). $\beta C$ (44-48) makes simultaneous backbone H-bonds with the strands A of the FnIII-3 and G2 of the FnIII-4, creating a continuous $\beta$-sheet that extends along the two FnIII domains. The $\beta$-strands of BP230 in the complex are stabilized by contacts with $\beta 4$. Yet, in the absence of $\beta 4$ the N-tail is highly sensitivity to controlled proteolysis (Figure S4), suggesting that this region of BP230 is mostly unstructured on its own.

The side chains of several residues of BP230 also contribute to the binding interface (Figure 3B-D). L32 and I41 are buried in hydrophobic pockets on the FnIII-4. S43, I45, and F47 dock in pockets formed at the interface of the two FnIII domains. V34 and L40 sit on shallower cavities on the surface of $\beta 4$. Most of the residues that bind in the pockets of $\beta 4$ are highly conserved in multiple species (Figures 3E and S5A). In addition, the side chains of N30 and N42 make H-bonds to the carbonyls of I1661 and F1566, respectively. In summary, binding is mainly driven by hydrophobic contacts, in accordance with an increase in the affinity of the interaction at high salt concentrations (Figure 3F).

To gain further insight of the determinants of the BP230-recognition by $\beta 4$, we applied a structure-based computational method to predict the BP230 sequences tolerated at the interface (Figures $3 \mathrm{G}$ and S5B). In addition to the residues that dock into the $\beta 4$ pockets, high preference is predicted for G37 and P38, which 
is in agreement with their conservation. Of these two residues, only P38 contacts $\beta 4$. Glycines appear frequently before prolines, where they facilitate the cis-trans isomerization of the latter, suggesting that the G37-P38 tandem might play an important role in the local rearrangement of the BP230 during binding to $\beta 4$. Noteworthy, BP180, Solo, and periaxin, which interact with the FnIII-3,4 region of $\beta 4$, do not have detectable sequence similarity with the tolerated binding motif.

The crystal structure is in good agreement with the mutagenesis data (Figures 3H and S6). R1463 in the FnIII-3 forms a salt bridge with E1659 in the FnIII-4 that closes over BP230. The inhibitory effect of the $\mathrm{R} 1463 \mathrm{E}$ and R1463A mutations suggests that this inter-domain clasp is essential to stabilize the interaction. A1468 is buried facing the $\beta 4$ inter-domain linker; an Arg or Asp at this position may clash with the linker altering the binding site. R1475, located near the inter-domain cleft that accommodates the strand $\beta C$ of BP230, does not engage directly with BP230, suggesting that the substitutions R1475E and R1475A affect indirectly the binding site. R1542 is near G48 of BP230; in addition, its side chain makes an H-bond with P1461 and contributes to the positioning of T1462, which in turn contacts BP230. Hence, the R1542E and R1542A substitutions are likely to alter this region of the interface. On the other hand, the nearby R1540 does not contact BP230, which explains the lack of effect on the binding of the R1540E and R1540A changes. P1576 in the FnIII-4 contacts L40 of BP230; the P1576R substitution is likely to reduce binding by distorting the interface. Finally, T1663 is near E29 of BP230. In the structure of the $\beta 4(T 1663 \mathrm{R})-\mathrm{BP} 230$ complex this engineered Arg makes an additional salt bridge with E29 without altering the conformation of E29 or the rest of BP230, which explains the increased affinity for BP230 of the T1663R mutant. On the other hand, the T1663D substitution is likely to reduce the affinity by creating an electrostatic repulsion with BP230.

Since mutations in $\beta 4$ that affect binding to BP230 are near the inter-domain interface, we analyzed their effect on the structure of the FnIII-3,4 using small angle x-ray scattering (SAXS) (Figure S7 and Table S3). Mutants R1463E, R1463A, R1475A, R1542E, R1542A, and T1663R behaved as the WT protein. The mutant R1475E showed slight differences with the WT protein, suggesting moderate self association. Only mutations A1468R and A1468D induced large deviations with respect to the WT $\beta 4$ fragment, which correspond to a large inter-domain flexibility. A1468 does contact BP230 directly. Thus, the deleterious effect of A1468R and A1468D on binding might be caused by the disruption of the inter-domain arrangement, suggesting that BP230 recognizes a pre-ordered surface in $\beta 4$.

\section{Binding of BP230 induces a conformational change in $\beta 4$}

Superimposition of the FnIII-3 domains in the structures of FnIII-3,4 bound to BP230 and in the free form, revealed differences in the relative orientation of the FnIII-4 domain (Figure 4A). The orientations of the 
FnIII-4 in the two states are related by a $\sim 38^{\circ}$ rotation around an axis located along the inter-domain interface.

To characterize the conformational change in $\beta 4$ induced by BP230, we used Double Electron-Electron Resonance (DEER) spectroscopy, a method that was useful to measure long range inter-domain distances between engineered paramagnetic groups in the FnIII-3,4 (Alonso-Garcia et al., 2015). We used $\beta 4$-FnIII3,4 mutants that contain the WT C1608 in the FnIII-4 and a second Cys introduced in the FnIII-3 at R1485C, R1504C, or L1497C (Figure 4B). Proteins were doubly labeled with the spin-probe MTSL and the distances between the pairs of paramagnetic groups were measured in the absence and in the presence of BP230 26-55 (Figure 4C,D). The distances in the free state matched those previously observed (Alonso-Garcia et al., 2015). In the presence of BP230, the peaks of the inter-spin distance distributions changed and were in agreement with the distances modeled in the $\beta 4$-BP230 complex. In summary, BP230 induces closing of the FnIII domains onto BP230, with the inter-domain linker acting as a hinge.

\section{The $\beta 4-B P 230$ interaction is required for the recruitment of BP230 into HD}

To assess the role of the binding interface on the recruitment of BP230 into HDs, we analyzed the distribution of BP230 and other HD proteins in PA-JEB keratinocytes (that do not express endogenous $\beta 4$ ) in which WT or point mutants of $\beta 4$ were expressed (Figure 5). Endogenous BP230 co-localized with WT $\beta 4$ and with endogenous BP180 and plectin, displaying a punctuated pattern characteristic of HDs. In contrast, BP230 had a diffused localization in PA-JEB keratinocytes expressing $\beta 4$ R1463E or R1463A, and BP230 did not co-localize either with BP180 or with plectin. $\beta 4$, BP180, and plectin showed a patched distribution in PA-JEB/ $\beta 4-\mathrm{R} 1463 \mathrm{E}$ or R1463A cells, which supports the view that BP230 is not required for the incorporation of these proteins into HDs (Koster et al., 2003; Wilhelmsen et al., 2006). When the high-affinity $\beta 4$ mutant T1663R was expressed, BP230 was again recruited into HDs and colocalized with $\beta 4$, BP180, and plectin. In summary, the interaction between the N-tail of BP230 and the FnIII-3,4 is a major determinant for the incorporation of BP230 into HDs in keratinocytes.

\section{Role of potentially phosphorylatable residues in the interaction}

Phosphorylation of $\beta 4$ by Ser/Thr kinases promotes HD disassembly by inhibiting the interaction of the $\alpha 6 \beta 4$ with plectin. This prompted us to analyze the role of potentially phosphorylatable residues in the binding of BP230 to $\beta 4$. The region FnIII-3,4 contains 21 predicted putative phosphorylatable Ser/Thr residues. Of those, only T1663 is in direct contact with BP230 (Figure S8A). As described above, the phosphomimetic change T1663D reduced the affinity for BP230 (Figure 2B,C). Another predicted phosphorylatable residue of $\beta 4, \mathrm{~S} 1556$, is located in the inter-domain linker and it was disordered in the structure. The sequence context of S1556 (PQSP) fits the optimal substrate sequence of the ERK1/2 
kinases (P-X-S/T-P), which are known to phosphorylate S1356 in the CS leading to the dissociation from plectin (Frijns et al., 2010). Yet, the substitution S1556D did not affect the affinity for BP230 with respect to WT $\beta 4$ (Figure S8B).

We also analyzed the role of potentially phosphorylatable residues in BP230 on the interaction with $\beta 4$ (Figure 6A-C). BP230 has six Ser/Thr residues in or near the $\beta 4$-binding site. The phosphomimetic substitutions S27D, S43D, S49D, and S51D did not affect the affinity for $\beta 4$. The substitution T39D resulted in a $\sim 3$-fold increase in the $K d$. Finally, the BP230 mutant S46D showed the weakest binding to $\beta 4$. Analysis of the effect of these point mutations on the interaction with $\beta 4$ using a pull-down assay (Figure 6C) showed that the BP230 mutants S27D, S43D, S49D, and S51D bound to GST- $\beta 4-C S-F n I I I-$ 3,4 similarly as the WT protein. Only a faint amount of the mutant T39D bound to $\beta 4$, and no interaction was observed for the mutant S46D. Thus, there is a good agreement between the results obtained with these two orthogonal methods.

S27, S49 and S51 are at the ends of the $\beta 4$-binding sequence. T39 is adjacent to the $\beta 4$ linker (Figure 6D); hence, the substitution T39D could disturb contacts with $\beta 4$. S43 docks in a polar pocket of $\beta 4$ that can accommodate an acidic group (Figure 6E). S46 is covered by the inter-domain salt bridge formed by R1463 and E1659 of $\beta 4$ (Figure 6F). The S46D substitution is likely to inhibit binding to $\beta 4$ by interfering with the R1463-E1659 salt bridge, which is in agreement with the inhibitory effect of the R1463A substitution. The mutant S46A showed slightly higher affinity for $\beta 4$ (Figure 6A-C); supporting the idea that the hydroxyl of S46 is not required for binding and that it imposes a penalty on the interaction. Collectively, our findings point to a potential role of T39 and S46 of BP230 and T1663 of $\beta 4$ in the regulation of the interaction.

\section{DISCUSSION}

Integrin $\alpha 6 \beta 4$ binds to plectin and BP230 through similar regions, formed by pairs of tandem FnIII domains, but these interactions occur through very distinct mechanisms. The FnIII-1,2 region of $\beta 4$ binds to a fairly flat surface on the ABD of plectin, which is a globular domain. In this study, we show that the FnIII-3,4 recognizes a linear motif in the N-tail of BP230, which has a relaxed structure in the free state. Our results suggest a mechanism for the high specificity and relatively low affinity of the $\beta 4-\mathrm{BP} 230$ complex. The extensive binding interface that includes specific contacts provides the basis for a selective interaction. On the other hand, binding is likely to cause a disorder-to-order transition of the N-tail of BP230, which would have an unfavorable contribution that would moderate the affinity (Dyson and Wright, 2005). The low affinity of the $\beta 4$-BP230 interaction is similar to that of the $\beta 4$-plectin complex $(K d \sim 30 \mu \mathrm{M})$. This supports the notion that HDs function as macromolecular Velcro® fasteners, in which 
firm attachment is achieved by combining multiple weak contacts in a process mediated by the presence of multiple binding sites within each protein, and the homo-association and clustering of HD proteins.

Incorporation of BP230 into HDs requires the previous interaction between plectin and $\alpha 6 \beta 4$, and the recruitment of BP180. This is depicted in a hierarchical model for the assembly of HDs (Koster et al., 2003; Wilhelmsen et al., 2006). The mechanisms for the recruitment of BP230 to HDs only at the final stage of assembly remained largely uncharacterized. BP230 interacts with the cytoplasmic domains of $\beta 4$ and BP180. The region 293-549 of BP230, which corresponds to the SR3-SR4-SH3-SR5 segment of its plakin domain, interacts with BP180, and this region of BP230 is required for the efficient recruitment into HDs (Koster et al., 2003). We have shown that point mutations in the FnIII-3,4 of $\beta 4$ that disrupt the BP230-binding interface and the direct interaction in vitro, also prevent the recruitment of BP230 to HDs in keratinocytes in culture. This suggests that binding to $\beta 4$, as observed in the $3 \mathrm{D}$ structure of the $\beta 4$ BP230 complex, is essential for the incorporation of BP230 in the HDs.

Besides providing additional binding sites, BP180 or plectin might favor the $\beta 4$-dependent recruitment of BP230 by regulating the affinity of the BP230- $\beta 4$ interaction (Figure 7). The BP230-binding site is accessible in the isolated FnIII-3,4 fragment, and binding of BP230 causes a closure of the FnIII-3 and FnIII-4 domains. In the full-length $\alpha 6 \beta 4$, the cytodomain of $\beta 4$ adopts a folded-over conformation in which the C-tail is positioned very close to the CS (Frijns et al., 2012), possibly through a direct intramolecular interaction between the CS and the C-tail (Koster et al., 2004; Rezniczek et al., 1998; Schaapveld et al., 1998). Given that the CS and C-tail flank the FnIII-3,4 region, this intramolecular clasp might mask the BP230-binding site in the FnIII-3,4 or might reduce the interdomain flexibility of $\beta 4$ impeding the conformational change linked to the binding of BP230. Plectin binds to $\beta 4$ at two sites. The ABD binds to the FnIII-1,2 and the beginning of the CS of $\beta 4$, and this interaction induces a reorganization of that part of the CS (de Pereda et al., 2009). In a second site of contact, the plakin domain of plectin binds to the CS and the C-tail of $\beta 4$. Thus, plectin binding to $\beta 4$ might alter the organization of the CS and C-tail, and as a consequence that of the FnIII-3,4. BP180 might also affect the FnIII-3,4 region through its interaction with the FnIII-3. In summary, plectin and BP180 could also favor allosterically the interaction between BP230 and $\beta 4$ by exposing the BP230-binding site or by reducing its rigidity.

The regulation of the interaction between $\beta 4$ and plectin, mainly through the phosphorylation of $\beta 4$ at specific Ser and Thr residues, plays a major role in the disassembly of HDs in keratinocytes. Phosphorylation of S1356 and S1364 in the CS prevents the association with the ABD of plectin, and phosphorylation of T1736 in the C-tail of $\beta 4$ reduces the interaction with the plakin domain of plectin (Frijns et al., 2012). It is unclear if other interactions in the HDs are directly inhibited during the disassembly, or if they are lost as a consequence of the disruption of the $\beta 4$-plectin complex. Phosphorylation of S1424 in the CS of $\beta 4$ is linked to a reduced association of $\beta 4$ with BP180 in 
migrating keratinocytes (Germain et al., 2009). S1424 is located outside the region of $\beta 4$ that interacts with BP180 (i.e. the FnIII-3) and it is not known if phosphorylation of S1424 reduces the affinity for BP180 directly. BP180 is also phosphorylated, probably at Ser/Thr, upon activation of PKC, which is linked to the dissociation of BP180 from HDs (Kitajima et al., 1999). Yet, it is not clear if the PKCmediated phosphorylation of BP180 disrupts its interactions with other hemidesmosomal proteins and it is required for the mobilization of BP180 from HDs, or if PKC mobilizes indirectly BP180 through the phosphorylation of $\beta 4$ and the disruption of the $\beta 4$-plectin interaction.

We have identified three potentially phosphorylatable residues, T1663 in $\beta 4$ and T39 and S46 in BP230, which are critical for the BP230- $\beta 4$ interaction. Substitution of these residues with Asp severely reduced the interaction. Of those, the strongest inhibition was observed for the S46D mutant of BP230. S46 is highly conserved and it is only replaced by Thr in coelacanths (Figure 3E). The conservation of S46 is striking because the hydroxyl group destabilizes, albeit moderately, the binding to $\beta 4$. This suggests that there has been a pressure to retain a Ser/Thr in this position. On the one hand, S46 could limit the affinity for $\beta 4$, which in turn could allow for an indirect regulation of the BP230- $\beta 4$ interaction during phases of HD assembly and disassembly. Alternatively, S46 might be a site for the direct regulation of the interaction. Phosphorylation of the N-tail of BP230 has not been described during HD disassembly; but its relaxed structure suggests that it might be a preferential region for post-translational modification. Since several kinases act on $\beta 4$ and BP180, it is reasonable that other proteins present in the HD niche might be phosphorylated as well. Future studies will be required to assess whether BP230 is post-translationally modified during the mobilization of hemidesmosomal proteins and whether active disruption of the BP230- $\beta 4$ interaction plays a role in the disassembly of HD.

Finally, our structural and mechanistic description of the $\beta 4$-BP230 interaction provides the basis to investigate its potential alteration in blistering diseases, and its regulation in normal keratinocytes during resting state, proliferation, and migration, and in invasive carcinoma cells.

\section{Acknowledgments}

We thank Prof Gunnar Jeschke for access to the EPR spectrometer. Lisa te Molder is thanked for assistance with image analysis and statistical analysis of data. We thank Constantina Bakolitsa for critical comments. We acknowledge ALBA-CELLS, Diamond Light Source (proposal MX10121), and the EMBL for access to synchrotron radiation facilities. This work was supported by the Spanish Ministry of Science, Innovation and Universities (grants BFU2009-08389, BFU2015-69499-P, and CTQ2015-64486-R) and Aragón local government by the action Grupo de Referencia "Biología Estructural"; these grants were cofunded by the European Regional Development Fund (ERDF). MGH, AC, and NAG were recipients of training grants from Universidad de Salamanca, Spanish Ministry of Education, Culture and Sport 
(FPU14/06259), and Consejo Superior de Investigaciones Científicas, respectively. This work received funding from the European Community's Seventh Framework Programme (FP7/2007-2013) under BioStruct-X (grant agreement $N^{\circ} 283570$ ). JMdP's institution is supported by the Programa de Apoyo a Planes Estratégicos de Investigación de Estructuras de Investigación de Excelencia co-funded by CastillaLeón autonomous government and ERDF (CLC-2017-01).

\section{Author contributions}

JAM, IGR, AS, JMdP, Conception and design, Acquisition of data, Analysis and interpretation of data, Writing the article; $\mathrm{MGH}, \mathrm{AC}$, Conception and design, Acquisition of data, Analysis and interpretation of data; NAG, MK, Acquisition of data, Analysis and interpretation of data.

\section{Declaration of interests}

The authors declare that no competing interests exist.

\section{FIGURES}

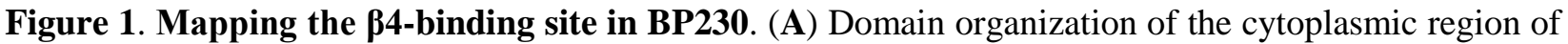
$\beta 4$ and the N-terminal region of BP230. (B) Equilibrium binding of $\beta 4-C S-F n I I I-3,4$ to Oregon Greenlabeled fragments of BP230, measured by fluorescence anisotropy $(r)$. Data are presented as fractional saturation. Lines are the fit of a one-to-one binding model. (C) Equilibrium binding of $\beta 4$-CS-FnIII- 3,4 to fluorescein-labeled peptides of BP230; data and fits are as in B. (D) Bar-chart with scatter plot of the $K d$ of the interaction between $\beta 4-\mathrm{CS}-$ FnIII-3,4 and the BP230 fragments (mean \pm SD). Asterisks indicate minimal values compatible with the data. See also Figure S1 and Table S1.

Figure 2. Mapping the BP230-binding site in $\boldsymbol{\beta 4}$. (A) Binding of fragments of $\beta 4$ to the fluoresceinlabeled BP230 peptide 26-55 measured by fluorescence anisotropy. Lines are the fit to the data. (B) Barchart of the $K d$ of the binding to BP230 26-55 of $\beta 4-\mathrm{CS}-\mathrm{Fn}-3,4$, WT and mutants. Error bars show the standard errors. Dashed vertical lines mark the $K d$ of WT $\beta 4$ (cyan) and the two-fold lower and higher values with respect to the WT (purple and red). (C) Binding of representative mutants. The binding curve of WT $\beta 4$ (dashed lines) is shown for comparison. (D) Surface representation of the unbound structure of $\beta 4$-FnIII-3,4. Residues are colored according to the effect of their mutations on the affinity for BP230, as indicated by the color bar. For residues changed in double and single mutants, the effect of the latter is shown. See also Table $\mathrm{S} 2$. 
Figure 3. Structure of BP230 bound to the FnIII-3,4 of $\beta 4$. (A) Two views of a ribbon representation of the structure of the WT $\beta 4-B P 230$ complex. (B) Close up of contacts between BP230 (blue) and $\beta 4$ (orange); waters are shown as red spheres. Polar contacts are shown as dashed lines. (C) Open-book view of the footprint of BP230 on the surface of the FnIII-4 (left) and the FnIII-3 and the liker (right) of $\beta 4$. (D) Schematic illustration of the BP230 residues that dock into pockets of $\beta 4$. (E) Alignment of the region 2655 of human BP230 with the sequences of representative species, colored by the evolutionary conservation. A multiple alignment of the sequences of 36 species used to calculate the conservation scores is shown in the Figure S5A. (F) Dependence of the $K d$ of the BP230- $\beta 4$ interaction on the salt concentration. (G) Sequence logo representation of the predicted consensus sequence that would tolerate binding to $\beta 4$. (H) Structure of the $\beta 4(\mathrm{WT})$-BP230 complex. BP230 is shown as a ribbon and $\beta 4$ is shown in a surface representation with residues colored according to the effect of their mutations. See also Figures S2 to S7 and Table S3.

Figure 4. BP230 induces a conformational change in the FnIII-3,4 of $\beta 4$. (A) Comparison of the structures of $\beta 4$ FnIII-3,4 in the free form (orange, SASBDB entry SASDAT6) (Alonso-Garcia et al., 2015 ) and bound to BP230 ( $\beta 4$ blue and BP230 magenta). Only the C $\alpha$ atoms of the FnIII-3 were used for the superimposition. The orientations of the FnIII-4 in the two structures are related by a rotation around a hinge axis (black line). (B) Worm models of the structures in A. The modeled average positions of the MTSL-paramagnetic centers (spheres) attached to the Cys pairs and the distances (dashed lines) in the two conformations are shown. (C) DEER normalized dipolar evolution (black lines) and fits to the data for the MTSL-labeled $\beta 4$ mutants in the absence (top) and in the presence of BP230 26-55 (bottom). (D) Interspin distance distributions calculated from the data in C.

Figure 5. Binding of BP230 N-tail to $\beta 4$ FnIII-3,4 is necessary for the recruitment of BP230 into HDs. (A) Confocal microscopy images of PA-JEB keratinocytes stably expressing $\beta 4 \mathrm{WT}$ or the mutants R1463E, R1463A, or T1663R. Cells were stained with antibodies against BP230 (green), $\beta 4$, BP180, or plectin (red), as indicated. Co-localization of BP230 with the other proteins appears as yellow. Nuclei were stained with DAPI (blue). Scale bars: $20 \mu \mathrm{m}$. (B) Quantification of the co-localization of $\beta 4$, plectin or BP180 with BP230. Manders' overlap coefficients (MOC) were calculated for at least 14 images per condition (14-72 images per condition from 1-3 experiments). Tukey box plots show the median (solid line), the $25^{\text {th }}$ and $75^{\text {th }}$ percentiles (boxes) for each distribution; whiskers represent 1.5 times above or below the interquartile range, outlier points outside the whiskers are displayed (dots). $* \mathrm{P}<0.0001$, unpaired, nonparametric Mann-Whitney test. 
Figure 6. Effect of phosphomimetic mutations in BP230 on the binding to $\beta 4$. (A) Binding of $\beta 4$-CSFnIII-3,4 to the BP230 10-162 fragment, WT and point mutants, measured by fluorescence anisotropy. Lines are the fits to the data. (B) Bar-chart with scatter plot of the $K d$ values of the interaction between $\beta 4$ and BP230 point mutants (mean \pm SD). (C) Pull-down analysis of the interaction between FLAG-BP230 (19-162), WT and point mutants, and GST- $\beta 4$ (1436-1666)-T1663R. The BP230 binding-deficient double mutant R1463E/T1663R was used as a negative control. BP230 proteins in the cell lysates (input) and in the bound samples (pull-down) were analyzed by WB with an anti-FLAG antibody. (D-F) Details of the structural environment of T39 (D), S43 (E) and S46 (F) of BP230 in the complex. See also Figure S8.

Figure 7. Model of regulation of BP230-\$4 interaction during HD assembly. Schematic representation of three steps during HD assembly. Recruitment of BP230 might require unlocking of the BP230-binding site in $\beta 4$, induced by plectin and/or BP180 binding to $\beta 4$, and a second interaction between BP230 and BP180. 
Table 1. Crystallographic data collection and refinement statistics

\begin{tabular}{|c|c|c|}
\hline Complex & $\beta 4(\mathrm{~T} 1663 \mathrm{R})-\mathrm{BP} 230$ & $\beta 4(\mathrm{WT})-\mathrm{BP} 230$ \\
\hline \multicolumn{3}{|l|}{ Data Collection } \\
\hline X-ray beamline & Xaloc (Alba) & i03 (Diamond) \\
\hline Space group & $\mathrm{C} 2$ & $\mathrm{C} 2$ \\
\hline Cell dimensions & $\begin{array}{l}\mathrm{a}=105.6 \AA \\
\mathrm{b}=59.5 \AA \\
\mathrm{c}=42.4 \AA \\
\beta=113.5^{\circ}\end{array}$ & $\begin{array}{l}\mathrm{a}=104.5 \AA \\
\mathrm{b}=60.7 \AA \\
\mathrm{c}=40.8 \AA \\
\beta=113.7^{\circ}\end{array}$ \\
\hline Wavelength $(\AA)$ & 0.97915 & 0.97625 \\
\hline Resolution $(\AA)$ & $1.55(1.59-1.55)^{\mathrm{a}}$ & $2.05(2.10-2.05)^{\mathrm{a}}$ \\
\hline Unique reflections & $35010(2562)^{\mathrm{a}}$ & $14766(1091)^{\mathrm{a}}$ \\
\hline Average multiplicity & $19.9(19.4)^{\mathrm{a}}$ & $6.7(6.9)^{\mathrm{a}}$ \\
\hline Completeness (\%) & $99.7(99.8)^{\mathrm{a}}$ & $99.5(100)^{\mathrm{a}}$ \\
\hline $\mathrm{R}_{\text {meas }}(\%)$ & $5.6(350)^{\mathrm{a}}$ & $10.5(195)^{\mathrm{a}}$ \\
\hline CC $1 / 2(\%)$ & $100(70.5)^{\mathrm{a}}$ & $99.8(69.4)^{\mathrm{a}}$ \\
\hline Mean $I / \sigma I$ & $26.4(1.53)^{\mathrm{a}}$ & $11.7(1.28)^{\mathrm{a}}$ \\
\hline \multicolumn{3}{|l|}{ Refinement } \\
\hline Resolution range $(\AA)$ & $39-1.55$ & $48-2.05$ \\
\hline Unique reflections, work/free & 33244 / 1749 & $14019 / 703$ \\
\hline $\mathrm{R}$ work $(\%)$ & 19.5 & 22.4 \\
\hline $\mathrm{R}$ free $^{\mathrm{b}}(\%)$ & 21.0 & 23.6 \\
\hline $\begin{array}{l}\text { Number of } \\
\text { residues ( } \beta 4 \text { / BP230) } \\
\text { waters } \\
\text { Glycerol }\end{array}$ & $\begin{array}{c}202 / 24 \\
105 \\
2\end{array}$ & $\begin{array}{c}199 / 23 \\
46 \\
-\end{array}$ \\
\hline $\begin{array}{l}\text { Average B value }\left(\AA^{2}\right) \\
\text { Wilson plot } \\
\text { Protein }(\beta 4 \text { / BP230) } \\
\text { Solvent } \\
\text { Glycerol }\end{array}$ & $\begin{array}{c}31.6 \\
44.4 / 42.5 \\
41.7 \\
51.3\end{array}$ & $\begin{array}{c}45.8 \\
64.3 / 63.2 \\
48.7 \\
-\end{array}$ \\
\hline rmsd bond lengths $(\AA)$ & 0.004 & 0.002 \\
\hline rmsd angles $\left({ }^{\circ}\right)$ & 0.721 & 0.496 \\
\hline $\begin{array}{l}\text { Ramachandran plot }^{\mathrm{c}} \text {, residues } \\
\text { Favored regions } \\
\text { Additionally allowed } \\
\text { Outliers }\end{array}$ & $\begin{array}{c}212(97.7 \%) \\
5(2.3 \%) \\
0\end{array}$ & $\begin{array}{c}213(98.2 \%) \\
4(1.8 \%) \\
0\end{array}$ \\
\hline PDB code & $6 \mathrm{GVK}$ & $6 \mathrm{GVL}$ \\
\hline
\end{tabular}

${ }^{a}$ Numbers in parenthesis correspond to the outer resolution shell.

${ }^{\mathrm{b}}$ Calculated using 5\% of reflections that were not included in the refinement.

${ }^{c}$ Analyzed with MOLPROBITY. 


\section{STAR METHODS}

\section{CONTACT FOR REAGENTS AND RESOURCES SHARING}

Further information and requests from resources and reagents should be directed to and will be fulfilled by the Lead Contact, Jose M de Pereda (pereda@usal.es)

\section{EXPERIMENTAL MODEL DETAILS}

E. coli strain BL21(DE3) was used for expression of recombinant proteins for in vitro studies, bacteria were grown in Terrific Broth medium.

The immortalized PA-JEB keratinocyte cell line, derived from cells isolated from a patient with pyloric atresia associated with junctional epidermolysis bullosa (PA-JEB), has been described previously (Schaapveld et al., 1998). PA-JEB keratinocytes were maintained in serum-free keratinocyte medium (SFM; Invitrogen) supplemented with $50 \mu \mathrm{g} / \mathrm{ml}$ bovine pituitary extract, $5 \mathrm{ng} / \mathrm{ml} \mathrm{EGF,} 100 \mathrm{U} / \mathrm{ml}$ penicillin, and $100 \mathrm{U} / \mathrm{ml}$ streptomycin. Human HEK293T cells (from embryo kidney) were grown in Dulbecco's modified Eagle's medium (DMEM) supplemented with $10 \%$ fetal bovine serum, $100 \mathrm{U} / \mathrm{ml}$ penicillin, $100 \mu \mathrm{g} / \mathrm{ml}$ streptomycin.

\section{METHOD DETAILS}

\section{Protein expression and purification}

The cDNA sequences coding for the fragments 1-162, 10-162, 19-162, 28-162, 32-162, 37-162, 41-162, 46-162, and 56-162 of human BP230 (Uniprot Q03001-8), and for the fragment 1436-1666 of human integrin $\beta 4$ (Uniprot P16144-2) were cloned into a modified pET15b vector (pETEV15b) (Alonso-García et al., 2009). Plasmids encoding for the $\beta 4$ fragments 1457-1666, 1457-1548, and 1572-1666 in pETEV15b were described earlier (Alonso-Garcia et al., 2015). The cDNA coding for a chimeric protein consisting of BP230 residues 19-58 and the SR2 of plectin, residues 420-530 (UniprotKB Q15149-2 was constructed by overlap extension PCR and was cloned in the pETEV15b vector as above. The cDNA of $\beta 4$ (1436-1666) was also subcloned into a pGEX-4T3 vector that was modified to have restriction sites compatible with pET15b. Point mutations were introduced by PCR using the QuikChange method. The correctness of all constructs was verified by DNA sequencing.

Proteins were expressed in E. coli strain BL21(DE3). The SR2 (56-162) of BP230 and the $\beta 4$ proteins were produced soluble and were purified by affinity chromatography as described (Manso et al., 2016). Other BP230 recombinant proteins were produced insoluble; they were purified under denaturing conditions and were refolded by rapid dilution as described (Alonso-García et al., 2009). The His-tag was 
cleaved by digestion with TEV (tobacco etch virus) protease, unless otherwise indicated. GST- $\beta 4$ (14361666) proteins were purified by affinity chromatography using a glutathione-agarose column.

\section{Peptides}

Peptides corresponding to the regions of BP230 26-46 (DSNENLLLVHCGPTLINSCISY, the C-terminal Tyr was included for quantification), 37-55 (GPTLINSCISFGSESFDGH), and 26-55 (DSNENLLLVHCGPTLINSCISFGSESFDGH) were custom synthesized labeled with N-terminal fluorescein (Thermo Fisher Scientific). Unlabeled peptide BP230 26-55 was obtained from Genosphere Biotechnologies.

\section{Labeling of proteins with Oregon Green 488}

BP230 proteins were labeled in thiol groups with Oregon Green 488 iodoacetamide (Invitrogen). Proteins at $250 \mu \mathrm{M}$ in $20 \mathrm{mM}$ Tris ( $\mathrm{pH} 8.0$ ), $150 \mathrm{mM} \mathrm{NaCl}$ were incubated overnight at $4{ }^{\circ} \mathrm{C}$ with a 10 -fold molar excess of the fluorescence probe. The reaction was stopped by adding a 10-fold molar excess of dithiothreitol (DTT) over the reagent and the proteins were separated from the unreacted probe by SEC using a Sephadex G25 (1 x $30 \mathrm{~cm})$ column.

\section{Fluorescence-based binding assay}

Labeled BP230 proteins and peptides at $1 \mu \mathrm{M}$ in $20 \mathrm{mM}$ Na-phosphate (pH 7.5), $150 \mathrm{mM} \mathrm{NaCl}, 1 \mathrm{mg} / \mathrm{ml}$ bovine serum albumin (BSA), unless otherwise indicated, were titrated with $\beta 4$-CS-FnIII- 3,4 . The fluorescence anisotropy of the probe was measured with a FluoroMax-3 spectrofluorometer (HORIBAJobin-Yvon) equipped with Glan-Thompson polarizers, using $496 \mathrm{~nm}$ excitation wavelength and collecting the emission at $518 \mathrm{~nm}$. Alternatively, anisotropy was measured in an Ultra Evolution plate reader (Tecan) using $485 \mathrm{~nm}$ and $535 \mathrm{~nm}$ excitation and emission filters, and 384-well black microplates (Greiner Bio-One). The $K d$ and the fluorescence anisotropy of the free $\left(r_{F}\right)$ and fully bound $\left(r_{B}\right)$ states, and their standard errors, were derived form the nonlinear square fitting of the following equation of a 1:1 binding model:

$$
\begin{gathered}
r=r_{F}+\left[\left(r_{B}-r_{F}\right)\left(\frac{\gamma-\sqrt{\gamma^{2}-4[\beta 4]_{\mathrm{T}}[\mathrm{BP} 230]_{\mathrm{T}}}}{2[\beta 4]_{\mathrm{T}}}\right)\right] \\
\gamma=K d+[\beta 4]_{\mathrm{T}}+[\mathrm{BP} 230]_{\mathrm{T}}
\end{gathered}
$$


Where, $[\mathrm{BP} 230]_{\mathrm{T}}$ is the total concentration of labeled-BP230, $[\beta 4]_{\mathrm{T}}$ is the total concentration of $\beta 4$ added, and $r$ is the observed fluorescence anisotropy of the probe. Fittings were performed using the Sigmaplot program.

Typically the highest useful concentration of $\beta 4$ attained in binding assays was below $250 \mu \mathrm{M}$, this resulted in reaching maximal binding saturation fraction $<0.9$, which limited the accuracy of the estimated $K d$. Thus, the $K d$ values were mainly used as relative measurements of the affinity. When only a small saturation fraction was obtained (e.g. low affinity mutants), the $r_{B}$ was fixed during the fitting using the value obtained for an interaction in which a high fractional saturation binding was obtained.

\section{Crystallization and determination of the structure of the $\beta 4-\mathrm{BP} 230$ complex}

Samples of the $\beta 4-F n I I I-3,4$ (1457-1666) mutant T1663R in complex with the peptide of BP230 (26-55) were prepared by adding $300 \mu \mathrm{M}$ of peptide from a $6 \mathrm{mM}$ stock in dimethyl sulfoxide (DMSO) to a 210 $\mu \mathrm{M}(5 \mathrm{mg} / \mathrm{ml})$ solution of $\beta 4-F n I I I-3,4$ in $10 \mathrm{mM}$ Tris ( $\mathrm{pH}$ 7.5), $100 \mathrm{mM} \mathrm{NaCl}, 2 \mathrm{mM}$ DTT. The sample was then extensively dialyzed against the same buffer to remove the DMSO using membranes of 0.5-1 $\mathrm{kDa}$ molecular weight cut-off. Crystals were obtained at room temperature by vapor diffusion mixing equal volumes of the $\beta 4-\mathrm{BP} 230$ complex at $2.5 \mathrm{mg} / \mathrm{ml}$ and crystallization solution. The best crystals were obtained using as crystallization solution $0.1 \mathrm{M}$ Na-acetate (pH 5.0), 20\% PEG 6000, $0.2 \mathrm{M} \mathrm{MgCl}_{2}$. Crystals were transferred to a similar solution containing $25 \%$ glycerol and were flashed-cooled in liquid nitrogen. Data were collected at $100 \mathrm{~K}$ on the Xaloc beam line of the ALBA synchrotron (Barcelona, Spain) (Juanhuix et al., 2014). A high multiplicity dataset was obtained by combining three sets of 1800 images, $0.2^{\circ}$ oscillation per image, measured at three positions of a single crystal. Diffraction data of these and all other crystals were processed with the XDS suite (Kabsch, 2010).

Crystals belong to the space group C2 (Table 1) and contain one copy of the complex in the asymmetric unit ( $46 \%$ solvent content). The structure was phased by molecular replacement with the program Phaser (McCoy et al., 2007) using the structures of the individual FnIII-3 and FnIII-4 domains (PDB codes 4WTW and 4WTX). The structure was refined against data extending to $1.55 \AA$ resolution with phenix.refine (Afonine et al., 2012), alternated with model building in Coot (Emsley et al., 2010). Refinement included overall anisotropic and bulk solvent corrections, positional refinement, restrained refinement of individual B-factor, and refinement of the translation/libration/screw-rotation (TLS) parameters of eight groups in $\beta 4$ and two in BP230. The refined model had excellent geometry and included residues 1457-1481, 1486-1552, and 1557-1665 of $\beta 4$, residues 27-50 of BP230, 104 molecules of water, and two molecules of glycerol. Detailed statistics of the refinement are shown in Table 1.

Samples of the WT $\beta 4-F n I I I-3,4$ in complex with BP230 26-55, were prepared as for the mutant. Crystals were obtained using as crystallization solution $0.1 \mathrm{M}$ phosphate-citrate (pH 4.2), 20\% PEG 8000, $0.2 \mathrm{M}$ 
$\mathrm{NaCl}$. Crystals were cryoprotected with $25 \%$ glycerol in the crystallization solution and were flashed cooled in liquid nitrogen. Diffraction data were collected at $100 \mathrm{~K}$ on the i03 beam line of the Diamond Light Source (Didcot, United Kingdom).

Crystals of the WT $\beta 4-B P 230$ complex were isomorphous with the $\beta 4(T 1663 R)-B P 230$ crystals. The structure was solved starting from the coordinates of the mutant complex and was refined against data extending to $2.05 \AA$. After rigid body fitting, the refinement was done as for the mutant, with the exception that only four TLS groups were refined. The refined model included residues 1457-1483, 14861551 , and 1561-1666 of $\beta 4$, residues 27-49 of BP230, and 46 molecules of water (Table 1).

\section{SAXS measurements and analysis}

SAXS data were measured at the P12 beamline of the European Molecular Biology Laboratory (EMBL) at the Deutsches Elektronen-Synchrotron (Hamburg, Germany) using radiation of wavelength ( $\lambda$ ) $1.24 \AA$ and a Pilatus 2M detector (Dectris) (Blanchet et al., 2015). Samples of $\beta 4-\mathrm{CS}-F n I I I-3,4$ WT and point mutants were equilibrated in $20 \mathrm{mM}$ Na-phosphate ( $\mathrm{pH} 7.5$ ), $150 \mathrm{mM} \mathrm{NaCl}, 5 \%$ glycerol, $3 \mathrm{mM}$ DTT. Samples at various concentrations were prepared by 2 -fold serial dilutions as indicated in the Table S3. SAXS data from protein samples and their buffers were measured consecutively at $10{ }^{\circ} \mathrm{C}$. Data were collected for a

range of the scattering-vector from 0.01 to $0.45 \AA^{-1}(q=(4 \pi \sin \theta) / \lambda$, where $2 \theta$ is the scattering angle). Data were processed and analyzed using the ATSAS package (Franke et al., 2017). Guinier analysis was done with the program AUTORG (Petoukhov et al., 2007). $P(r)$ functions were calculated using data to $q \leq 0.30$ $\AA^{-1}$ with the program GNOM (Svergun, 1992). Inter-domain flexibility was analyzed using the Ensemble Optimization Method with the program EOM 2.1 (Tria et al., 2015). First, a pool of 15.000 theoretical conformers that represent the potential conformation diversity of the $\beta 4$-CS-FnIII-3,4 was generated treating the FnIII domains as rigid bodies and the CS and the linker as flexible regions. Then, combinations of the theoretical scattering profiles of the models were fitted to the experimental curves.

\section{Site-directed spin labeling and DEER measurements and analysis}

The mutant proteins of $\beta 4-F n I I I-3,4$ containing two cysteines R1485C/C1608, L1497C/C1608, and $\mathrm{R} 1504 \mathrm{C} / \mathrm{C} 1608$, all of which also carried the changes C1559A/C1483S/T1663R, were labeled with the thiol-reactive paramagnetic probe $S$-(1-oxyl-2,2,5,5-tetramethyl-2,5-dihydro-1H-pyrrol-3-yl)methyl methanesulfonothioate (MTSL) as described (Alonso-Garcia et al., 2015). Solutions of MTSL-labeled proteins between 70 to $100 \mu \mathrm{M}$ in $20 \mathrm{mM}$ Na-phosphate (pH 7.5), $150 \mathrm{mM} \mathrm{NaCl}$, without or with $\sim 5$-fold molar excess of BP230 26-55, were mixed with deuterated glycerol in a 2:1 ratio to obtain a vitrified solution with longer transverse relaxation times upon freezing; they were subsequently transferred into 3 $\mathrm{mm}$ OD quartz tubes and stored in liquid nitrogen until measurement. 
DEER measurements were performed at a temperature of $50 \mathrm{~K}$ in a Q-band ( $34 \mathrm{GHz})$ home-made EPR spectrometer (Gromov et al., 2001) equipped with a rectangular TE(102) cavity allowing for big sample volumes (Tschaggelar et al., 2009). The two-frequency 4-pulse DEER sequence (Pannier et al., 2000) was used setting the pump pulses at the maximum of the nitroxide spectrum. The DEER traces were processed with DeerAnalysis, an ad hoc toolbox programmed for MATLAB (Jeschke et al., 2006) and analyzed using a model-free Tikhonov regularization implemented in the same software to obtain the distance distributions.

\section{Structure and sequence analysis}

Domain motions that relate the BP230-bound and the free structures of $\beta 4$-FnIII-3,4 were analyzed with the program DynDom (Hayward and Berendsen, 1998). Evolutionary conservation scores were calculated using the Consurf Server (Ashkenazy et al., 2016). Prediction of the sequence tolerance within the $\beta 4$ binding site of BP230 was done using the Rosetta Sequence Tolerance method (Smith and Kortemme, 2011). Molecular figures were created with PyMOL (Schrödinger, 2015). Analysis of potentially phosphorylatable sites was done using the Phosphonet server (Kinexus Bioinformatics) (http://www.phosphonet.ca/).

\section{Antibodies}

The following primary antibodies were used: mouse monoclonal antibody (mAb) 450-11A against $\beta 4$ (BD Biosciences), human mAb 5E against BP230 (Ishiko et al., 1993), mouse mAb 233 against BP180 (Nishizawa et al., 1993), guinea pig polyclonal antibody (pAb) P1 against plectin (Stegh et al., 2000), and rabbit pAb D-8 against FLAG (Santa Cruz Biotechnology). Secondary antibodies were as follows: goat anti-mouse Texas Red, goat anti-human Alexa Fluor 488, goat anti-guinea pig Alexa Fluor 488 (Thermo Fisher Scientific), and donkey anti-human Texas Red (Jackson ImmunoResearch).

\section{cDNA constructs for expression in mammalian cell cultures}

Point mutations R1463E, R1463A, C1559A, and T1663R were introduced by site directed mutagenesis (see above) in a construct coding full-length $\beta 4$ in the pUC18 vector (Niessen et al., 1997b). Retroviral vectors with mutant $\beta 4$ cDNAs were generated by subcloning the mutant $\beta 4$ cDNAs in the LZRS-MSIRES-ZEO vector as described (Frijns et al., 2012).

The cDNA coding for the region 19-162 of BP230 was amplified by PCR using primers that added EcoRI and NotI sites at each end, respectively. The PCR product was digested and was cloned using the same sites in the pCEF-FLAG vector (Chiariello et al., 2000), which codes for an N-terminal FLAG tag. Point mutations were introduced in this construct by site-directed mutagenesis. 


\section{Cell culture and immunofluorescence}

PA-JEB/ $\beta 4$ keratinocytes stably expressing WT or point mutants of $\beta 4$ were generated by retroviral transduction (Sterk et al., 2000). Full-length and mutant $\beta 4$ A constructs in LZRS retroviral vector (Kinsella and Nolan, 1996) were introduced into Phoenix packaging cells by the calcium phosphate precipitation method and virus containing supernatant was collected (Geuijen and Sonnenberg, 2002). PAJEB cells were infected with the recombinant virus in the presence of $8 \mu \mathrm{g} / \mathrm{ml}$ polybrene (hexadimethrine bromide, Sigma). After incubation for $16 \mathrm{~h}$ at $37^{\circ} \mathrm{C}$, infected cells were selected with $0.2 \mathrm{mg} / \mathrm{ml}$ zeocin (Invitrogen). Cells expressing $\alpha 6 \beta 4$ at their surface were isolated by FACS $®$, expanded, and analyzed.

For immunostaining, PA-JEB/ $\beta 4$ cells were seeded on glass coverslips and cultured for $24 \mathrm{~h}$ in complete $\mathrm{KGM}$, and then continued to be cultured for a further $16 \mathrm{~h}$ in DMEM with $10 \%$ FCS. Cells were fixed in $1 \%$ paraformaldehyde in PBS, permeabilized with $0.2 \%$ Triton X-100 in PBS for 5 min, blocked with 2\% BSA in PBS, and subsequently incubated for $1 \mathrm{~h}$ with the following primary antibodies: 5E anti BP230 (dilution 1:500), 450-11A against $\beta 4$ (dilution 1:500), P1 against plectin (dilution 1:200), and 233 against BP180 (undiluted). After washing three times with PBS, the cells were incubated with the secondary antibodies for $1 \mathrm{~h}$. The cells were then washed three times with PBS, and nuclei were stained with 4',6diamidino-2-phenylindole (DAPI, Sigma),. Finally, the coverslips were mounted onto glass slides in Mowiol-DABCO and observed using a Leica TCS SP5 confocal microscope.

\section{Pull-down assay}

HEK293T cells were transfected with pCEF-FLAG-BP230 (19-162) constructs using polyethylenimine (PEI) (PEI:DNA ratio 2:1). $48 \mathrm{~h}$ after transfection, cells from a confluent 10-cm plate were lysed in $600 \mu \mathrm{l}$ lysis buffer consisting of $20 \mathrm{mM}$ Tris ( $\mathrm{pH} 7.5$ ), $500 \mathrm{mM} \mathrm{NaCl}, 0.5 \%$ Triton X-100, $1 \mathrm{mM} \mathrm{Na}_{3} \mathrm{VO}_{4}, 25$ $\mathrm{mM} \mathrm{NaF}, 1 \mathrm{mM}$ PMSF, and protease inhibitor cocktail (Roche). Lysates were cleared by centrifugation at $16000 \mathrm{x} g$ for $15 \mathrm{~min}$ at $4{ }^{\circ} \mathrm{C}$. Supernatants were incubated for 1 hour at $4{ }^{\circ} \mathrm{C}$ with 0.6 nmoles of the GST$\beta 4$ (1436-1666) T1663R or the inactive double mutant R1463E/T1663R and $20 \mu$ of glutathione-agarose resin (Agarose Bead Technologies). The resin was washed four times with lysis buffer and the bound proteins were extracted with SDS-PAGE sample buffer. Bound FLAG-BP230 proteins were analyzed by immunobloting using anti-FLAG antibody (dilution 1:1000) and as secondary antibody a goat anti-rabbit IgG DyLight 800 (Thermo Fisher Scientific) (dilution 1:5000). Results were detected by infrared fluorescence using an Odyssey imaging system (Li-Cor).

\section{QUANTIFICATION AND STATISTICAL ANALYSIS}


In case of single fluorescence anisotropy titrations, the asymptotic standard errors of the $K d$ were derived from the nonlinear least square fits of the respective binding isotherms. Alternatively, when multiple titrations were performed, data is presented as mean \pm standard deviation (SD).

The degree of co-localization between BP230 and $\beta 4$, plectin or BP180 in immunofluorescence confocal microscopy images was quantified using the Manders' overlap coefficients (MOC) calculated with the JACoP plugin from Image-J (Bolte and Cordelieres, 2006). Statistical significance was analysed using unpaired, nonparametric Mann-Whitney test in GraphPad Prism.

\section{DATA AVAILABILITY}

Coordinates and structure factors have been deposited to the Protein Data Bank (PDB) under the accession numbers 6GVL ( $\beta 4(\mathrm{WT})$-BP230) and 6GVK ( $\beta 4(\mathrm{~T} 1663 \mathrm{R})$-BP230); raw diffraction images have been deposited in the Zenodo repository (DOI 10.5281/zenodo.1287191 and 10.5281/zenodo.1286853). SAXS data have been deposited in the Small Angle Scattering Biological Data Bank (SASBDB) under codes SASDDE8, SASDDF8, SASDDG8, SASDDH8, SASDDJ8, SASDDK8, SASDDL8, SASDDM8, SASDDN8, and SASDDP8.

\section{REFERENCES}

Afonine, P.V., Grosse-Kunstleve, R.W., Echols, N., Headd, J.J., Moriarty, N.W., Mustyakimov, M., Terwilliger, T.C., Urzhumtsev, A., Zwart, P.H., and Adams, P.D. (2012). Towards automated crystallographic structure refinement with phenix.refine. Acta Crystallogr D Biol Crystallogr 68, 352-367.

Alonso-Garcia, N., Garcia-Rubio, I., Manso, J.A., Buey, R.M., Urien, H., Sonnenberg, A., Jeschke, G., and de Pereda, J.M. (2015). Combination of X-ray crystallography, SAXS and DEER to obtain the structure of the FnIII3,4 domains of integrin alpha6beta4. Acta Crystallogr D Biol Crystallogr 71, 969-985.

Alonso-García, N., Ingles-Prieto, A., Sonnenberg, A., and De Pereda, J.M. (2009). Structure of the Calx-beta domain of the integrin beta4 subunit: insights into function and cation-independent stability. Acta Crystallogr D Biol Crystallogr 65, 858-871.

Ashkenazy, H., Abadi, S., Martz, E., Chay, O., Mayrose, I., Pupko, T., and Ben-Tal, N. (2016). ConSurf 2016: an improved methodology to estimate and visualize evolutionary conservation in macromolecules. Nucleic Acids Res 44, W344-350.

Blanchet, C.E., Spilotros, A., Schwemmer, F., Graewert, M.A., Kikhney, A., Jeffries, C.M., Franke, D., Mark, D., Zengerle, R., Cipriani, F., et al. (2015). Versatile sample environments and automation for biological solution Xray scattering experiments at the P12 beamline (PETRA III, DESY). J Appl Cryst 48, 431-443.

Bolte, S., and Cordelieres, F.P. (2006). A guided tour into subcellular colocalization analysis in light microscopy. Journal of microscopy 224, 213-232. 
Chiariello, M., Marinissen, M.J., and Gutkind, J.S. (2000). Multiple mitogen-activated protein kinase signaling pathways connect the cot oncoprotein to the c-jun promoter and to cellular transformation. Mol Cell Biol 20, 1747-1758.

de Pereda, J.M., Lillo, M.P., and Sonnenberg, A. (2009). Structural basis of the interaction between integrin alpha6beta4 and plectin at the hemidesmosomes. EMBO J 28, 1180-1190.

Dyson, H.J., and Wright, P.E. (2005). Intrinsically unstructured proteins and their functions. Nat Rev Mol Cell Biol 6, 197-208.

Emsley, P., Lohkamp, B., Scott, W.G., and Cowtan, K. (2010). Features and development of Coot. Acta Crystallogr D Biol Crystallogr 66, 486-501.

Franke, D., Petoukhov, M.V., Konarev, P.V., Panjkovich, A., Tuukkanen, A., Mertens, H.D.T., Kikhney, A.G., Hajizadeh, N.R., Franklin, J.M., Jeffries, C.M., and Svergun, D.I. (2017). ATSAS 2.8: a comprehensive data analysis suite for small-angle scattering from macromolecular solutions. J Appl Cryst 50, 1212-1225.

Frijns, E., Kuikman, I., Litjens, S., Raspe, M., Jalink, K., Ports, M., Wilhelmsen, K., and Sonnenberg, A. (2012). Phosphorylation of threonine 1736 in the C-terminal tail of integrin beta4 contributes to hemidesmosome disassembly. Mol Biol Cell 23, 1475-1485.

Frijns, E., Sachs, N., Kreft, M., Wilhelmsen, K., and Sonnenberg, A. (2010). EGF-induced MAPK signaling inhibits hemidesmosome formation through phosphorylation of the integrin \{beta\}4. J Biol Chem 285, 37650-37662.

Fujiwara, S., Matsui, T.S., Ohashi, K., Deguchi, S., and Mizuno, K. (2018). Solo, a RhoA-targeting guanine nucleotide exchange factor, is critical for hemidesmosome formation and acinar development in epithelial cells. PloS one 13, e0195124.

Geerts, D., Fontao, L., Nievers, M.G., Schaapveld, R.Q., Purkis, P.E., Wheeler, G.N., Lane, E.B., Leigh, I.M., and Sonnenberg, A. (1999). Binding of integrin alpha6beta4 to plectin prevents plectin association with F-actin but does not interfere with intermediate filament binding. J Cell Biol 147, 417-434.

Germain, E.C., Santos, T.M., and Rabinovitz, I. (2009). Phosphorylation of a novel site on the $\{$ beta 4 integrin at the trailing edge of migrating cells promotes hemidesmosome disassembly. Mol Biol Cell 20, 56-67.

Geuijen, C.A., and Sonnenberg, A. (2002). Dynamics of the alpha6beta4 integrin in keratinocytes. Mol Biol Cell 13, 3845-3858.

Gipson, I.K., Spurr-Michaud, S., Tisdale, A., Elwell, J., and Stepp, M.A. (1993). Redistribution of the hemidesmosome components alpha 6 beta 4 integrin and bullous pemphigoid antigens during epithelial wound healing. Exp Cell Res 207, 86-98.

Gromov, I., Shane, J., Forrer, J., Rakhmatoullin, R., Rozentzwaig, Y., and Schweiger, A. (2001). A Q-band pulse EPR/ENDOR spectrometer and the implementation of advanced one- and two-dimensional pulse EPR methodology. J Magn Reson. 149, 196-203.

Groves, R.W., Liu, L., Dopping-Hepenstal, P.J., Markus, H.S., Lovell, P.A., Ozoemena, L., Lai-Cheong, J.E., Gawler, J., Owaribe, K., Hashimoto, T., et al. (2010). A homozygous nonsense mutation within the dystonin gene coding for the coiled-coil domain of the epithelial isoform of BPAG1 underlies a new subtype of autosomal recessive epidermolysis bullosa simplex. J Invest Dermatol 130, 1551-1557. 
Guo, L., Degenstein, L., Dowling, J., Yu, Q.C., Wollmann, R., Perman, B., and Fuchs, E. (1995). Gene targeting of BPAG1: abnormalities in mechanical strength and cell migration in stratified epithelia and neurologic degeneration. Cell 81, 233-243.

Hayward, S., and Berendsen, H.J. (1998). Systematic analysis of domain motions in proteins from conformational change: new results on citrate synthase and T4 lysozyme. Proteins 30, 144-154.

Herold-Mende, C., Kartenbeck, J., Tomakidi, P., and Bosch, F.X. (2001). Metastatic growth of squamous cell carcinomas is correlated with upregulation and redistribution of hemidesmosomal components. Cell and tissue research 306, 399-408.

Hopkinson, S.B., and Jones, J.C. (2000). The N terminus of the transmembrane protein BP180 interacts with the Nterminal domain of BP230, thereby mediating keratin cytoskeleton anchorage to the cell surface at the site of the hemidesmosome. Mol Biol Cell 11, 277-286.

Ishiko, A., Shimizu, H., Kikuchi, A., Ebihara, T., Hashimoto, T., and Nishikawa, T. (1993). Human autoantibodies against the 230-kD bullous pemphigoid antigen (BPAG1) bind only to the intracellular domain of the hemidesmosome, whereas those against the $180-\mathrm{kD}$ bullous pemphigoid antigen (BPAG2) bind along the plasma membrane of the hemidesmosome in normal human and swine skin. The Journal of clinical investigation 91 , 1608-1615.

Jefferson, J.J., Ciatto, C., Shapiro, L., and Liem, R.K. (2007). Structural analysis of the plakin domain of bullous pemphigoid antigen1 (BPAG1) suggests that plakins are members of the spectrin superfamily. J Mol Biol 366 , 244-257.

Jeschke, G., Chechik, V., Ionita, P., Godt, A., Zimmermann, H., Banham, J., Timmel, C.R., Hilger, D., and Jung, H. (2006). DeerAnalysis2006 - a comprehensive software package for analyzing pulsed ELDOR data. Appl Magn Reson 30, 473-498.

Juanhuix, J., Gil-Ortiz, F., Cuni, G., Colldelram, C., Nicolas, J., Lidon, J., Boter, E., Ruget, C., Ferrer, S., and Benach, J. (2014). Developments in optics and performance at BL13-XALOC, the macromolecular crystallography beamline at the ALBA synchrotron. J Synchrotron Radiat 21, 679-689.

Kabsch, W. (2010). Xds. Acta Crystallogr D Biol Crystallogr 66, 125-132.

Kinsella, T.M., and Nolan, G.P. (1996). Episomal vectors rapidly and stably produce high-titer recombinant retrovirus. Human gene therapy 7, 1405-1413.

Kitajima, Y., Aoyama, Y., and Seishima, M. (1999). Transmembrane signaling for adhesive regulation of desmosomes and hemidesmosomes, and for cell-cell datachment induced by pemphigus IgG in cultured keratinocytes: involvement of protein kinase C. The journal of investigative dermatology. Symposium proceedings $4,137-144$.

Koster, J., Geerts, D., Favre, B., Borradori, L., and Sonnenberg, A. (2003). Analysis of the interactions between BP180, BP230, plectin and the integrin alpha6beta4 important for hemidesmosome assembly. J Cell Sci 116 , 387-399.

Koster, J., van Wilpe, S., Kuikman, I., Litjens, S.H., and Sonnenberg, A. (2004). Role of binding of plectin to the integrin beta4 subunit in the assembly of hemidesmosomes. Mol Biol Cell 15, 1211-1223. 
Leung, C.L., Zheng, M., Prater, S.M., and Liem, R.K. (2001). The BPAG1 locus: Alternative splicing produces multiple isoforms with distinct cytoskeletal linker domains, including predominant isoforms in neurons and muscles. J Cell Biol 154, 691-697.

Manso, J.A., Garcia Rubio, I., Gomez-Hernandez, M., Ortega, E., Buey, R.M., Carballido, A.M., Carabias, A., Alonso-Garcia, N., and de Pereda, J.M. (2016). Purification and Structural Analysis of Plectin and BPAG1e. Methods Enzymol 569, 177-196.

Margadant, C., Frijns, E., Wilhelmsen, K., and Sonnenberg, A. (2008). Regulation of hemidesmosome disassembly by growth factor receptors. Curr Opin Cell Biol 20, 589-596.

McCoy, A.J., Grosse-Kunstleve, R.W., Adams, P.D., Winn, M.D., Storoni, L.C., and Read, R.J. (2007). Phaser crystallographic software. J Appl Cryst 40, 658-674.

Niessen, C.M., Hulsman, E.H., Oomen, L.C., Kuikman, I., and Sonnenberg, A. (1997a). A minimal region on the integrin beta4 subunit that is critical to its localization in hemidesmosomes regulates the distribution of HD1/plectin in COS-7 cells. J Cell Sci 110, 1705-1716.

Niessen, C.M., Hulsman, E.H., Rots, E.S., Sanchez-Aparicio, P., and Sonnenberg, A. (1997b). Integrin alpha 6 beta 4 forms a complex with the cytoskeletal protein HD1 and induces its redistribution in transfected COS-7 cells. Mol Biol Cell 8, 555-566.

Nishizawa, Y., Uematsu, J., and Owaribe, K. (1993). HD4, a $180 \mathrm{kDa}$ bullous pemphigoid antigen, is a major transmembrane glycoprotein of the hemidesmosome. Journal of biochemistry 113, 493-501.

Pannier, M., Veit, S., Godt, A., Jeschke, G., and Spiess, H.W. (2000). Dead-time free measurement of dipole-dipole interactions between electron spins. Journal of magnetic resonance 142, 331-340.

Petoukhov, M.V., Konarev, P.V., Kikhney, A.G., and Svergun, D.I. (2007). ATSAS 2.1 - towards automated and web-supported small-angle scattering data analysis. J Appl Cryst 40, s223-s228.

Raasakka, A., Linxweiler, H., Brophy, P.J., Sherman, D.L., and Kursula, P. (2019). Direct binding of the flexible Cterminal segment of periaxin to $\beta 4$ integrin suggests a molecular basis for CMT4F. bioRxiv, 524793.

Rabinovitz, I., Toker, A., and Mercurio, A.M. (1999). Protein kinase C-dependent mobilization of the alpha6beta4 integrin from hemidesmosomes and its association with actin-rich cell protrusions drive the chemotactic migration of carcinoma cells. J Cell Biol 146, 1147-1160.

Rabinovitz, I., Tsomo, L., and Mercurio, A.M. (2004). Protein kinase C-alpha phosphorylation of specific serines in the connecting segment of the beta 4 integrin regulates the dynamics of type II hemidesmosomes. Mol Cell Biol 24, 4351-4360.

Rezniczek, G.A., de Pereda, J.M., Reipert, S., and Wiche, G. (1998). Linking integrin alpha6beta4-based cell adhesion to the intermediate filament cytoskeleton: direct interaction between the beta4 subunit and plectin at multiple molecular sites. J Cell Biol 141, 209-225.

Schaapveld, R.Q., Borradori, L., Geerts, D., van Leusden, M.R., Kuikman, I., Nievers, M.G., Niessen, C.M., Steenbergen, R.D., Snijders, P.J., and Sonnenberg, A. (1998). Hemidesmosome formation is initiated by the beta4 integrin subunit, requires complex formation of beta4 and HD1/plectin, and involves a direct interaction between beta4 and the bullous pemphigoid antigen 180. J Cell Biol 142, 271-284.

Schrödinger, L. (2015). The PyMOL Molecular Graphics System, Version 1.8. 
Smith, C.A., and Kortemme, T. (2011). Predicting the tolerated sequences for proteins and protein interfaces using RosettaBackrub flexible backbone design. PloS one 6, e20451.

Song, J.G., Kostan, J., Drepper, F., Knapp, B., de Almeida Ribeiro, E., Jr., Konarev, P.V., Grishkovskaya, I., Wiche, G., Gregor, M., Svergun, D.I., et al. (2015). Structural Insights into Ca(2+)-Calmodulin Regulation of Plectin 1aIntegrin beta4 Interaction in Hemidesmosomes. Structure 23, 558-570.

Sonnenberg, A., Rojas, A.M., and de Pereda, J.M. (2007). The structure of a tandem pair of spectrin repeats of plectin reveals a modular organization of the plakin domain. J Mol Biol 368, 1379-1391.

Stegh, A.H., Herrmann, H., Lampel, S., Weisenberger, D., Andra, K., Seper, M., Wiche, G., Krammer, P.H., and Peter, M.E. (2000). Identification of the cytolinker plectin as a major early in vivo substrate for caspase 8 during CD95- and tumor necrosis factor receptor-mediated apoptosis. Mol Cell Biol 20, 5665-5679.

Sterk, L.M., Geuijen, C.A., Oomen, L.C., Calafat, J., Janssen, H., and Sonnenberg, A. (2000). The tetraspan molecule CD151, a novel constituent of hemidesmosomes, associates with the integrin alpha6beta4 and may regulate the spatial organization of hemidesmosomes. J Cell Biol 149, 969-982.

Svergun, D. (1992). Determination of the regularization parameter in indirect-transform methods using perceptual criteria. J Appl Cryst 25, 495-503.

Tria, G., Mertens, H.D.T., Kachala, M., and Svergun, D.I. (2015). Advanced ensemble modelling of flexible macromolecules using X-ray solution scattering. IUCrJ 2, 207-217.

Tschaggelar, R., Kasumaj, B., Santangelo, M.G., Forrer, J., Leger, P., Dube, H., Diederich, F., Harmer, J., Schuhmann, R., Garcia-Rubio, I., and Jeschke, G. (2009). Cryogenic 35GHz pulse ENDOR probehead accommodating large sample sizes: Performance and applications. J Magn Reson. 200, 81-87.

Uematsu, J., Nishizawa, Y., Sonnenberg, A., and Owaribe, K. (1994). Demonstration of type II hemidesmosomes in a mammary gland epithelial cell line, BMGE-H. Journal of biochemistry 115, 469-476.

Van den Bergh, F., Eliason, S.L., and Giudice, G.J. (2011). Type XVII collagen (BP180) can function as a cellmatrix adhesion molecule via binding to laminin 332. Matrix Biol 30, 100-108.

Walko, G., Castanon, M.J., and Wiche, G. (2015). Molecular architecture and function of the hemidesmosome. Cell and tissue research 360, 529-544.

Wilhelmsen, K., Litjens, S.H., Kuikman, I., Margadant, C., van Rheenen, J., and Sonnenberg, A. (2007). Serine phosphorylation of the integrin beta4 subunit is necessary for epidermal growth factor receptor induced hemidesmosome disruption. Mol Biol Cell 18, 3512-3522.

Wilhelmsen, K., Litjens, S.H., and Sonnenberg, A. (2006). Multiple functions of the integrin alpha6beta4 in epidermal homeostasis and tumorigenesis. Mol Cell Biol 26, 2877-2886. 
Fig 1
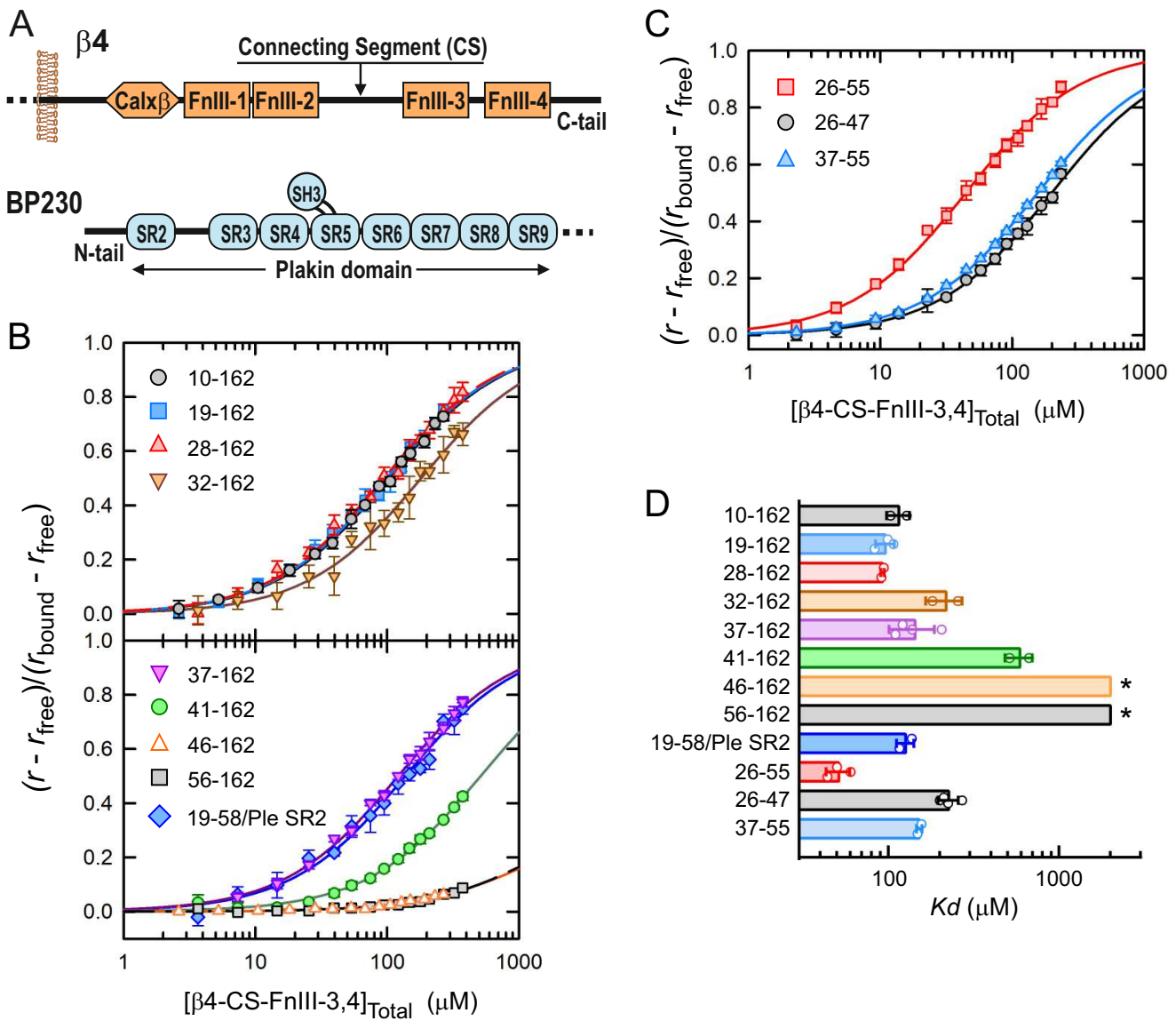
Fig 2

A

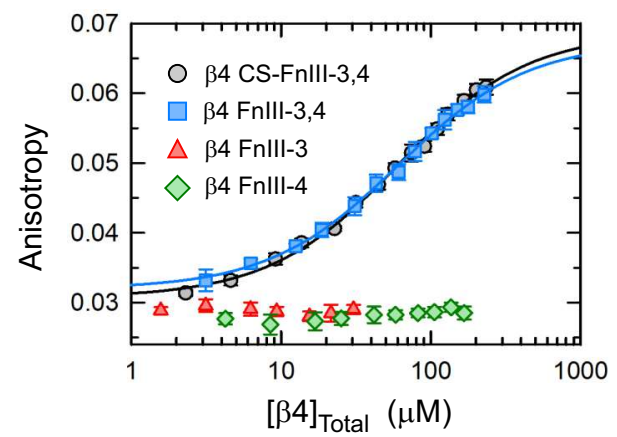

C
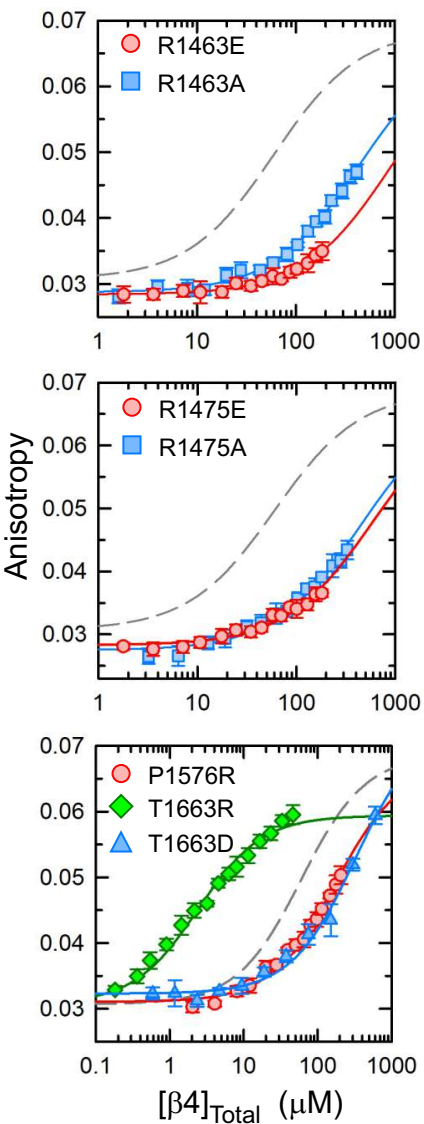

B
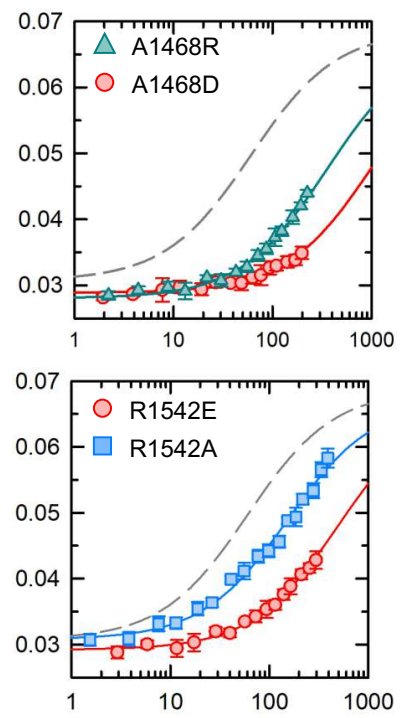

$[\beta 4]_{\text {Total }}(\mu \mathrm{M})$

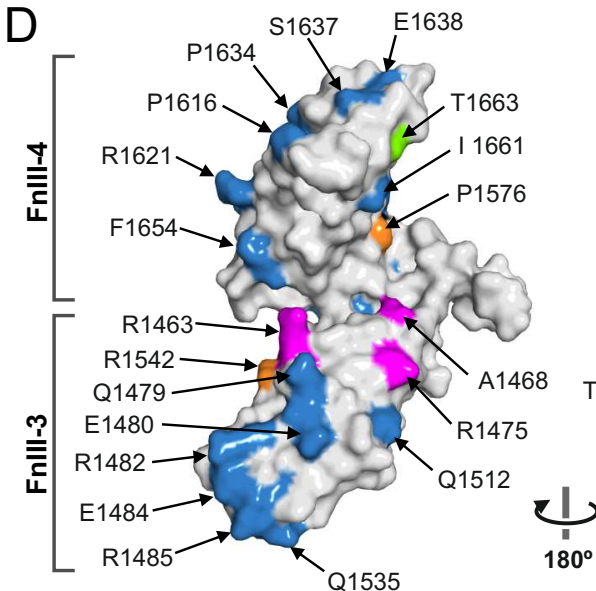

Concave side
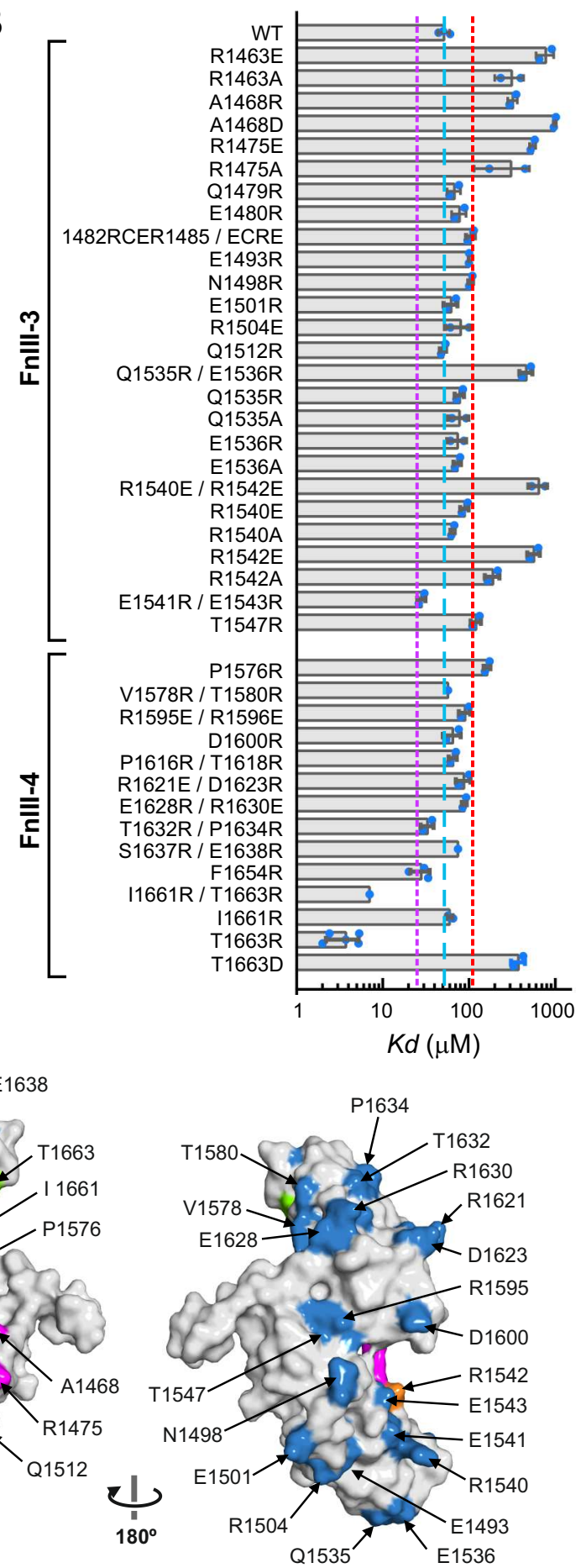

Convex side

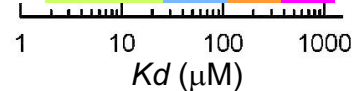


Fig 3

A

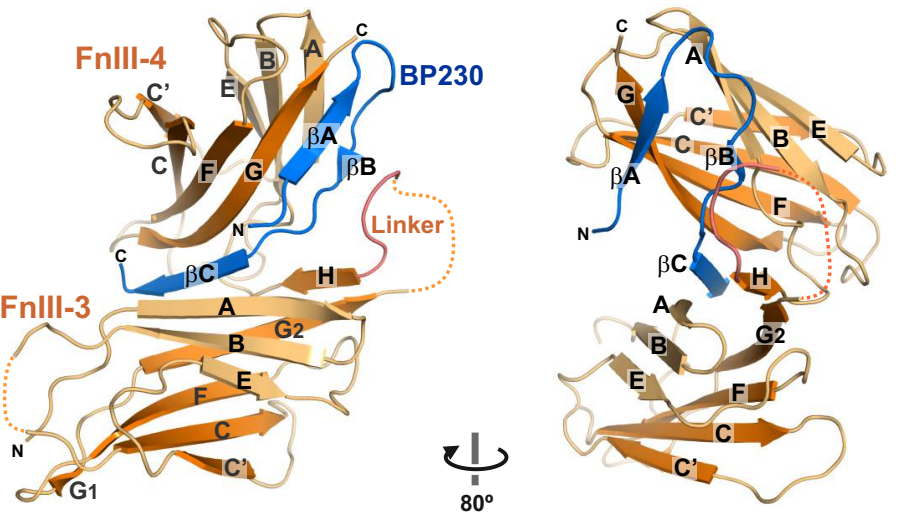

C

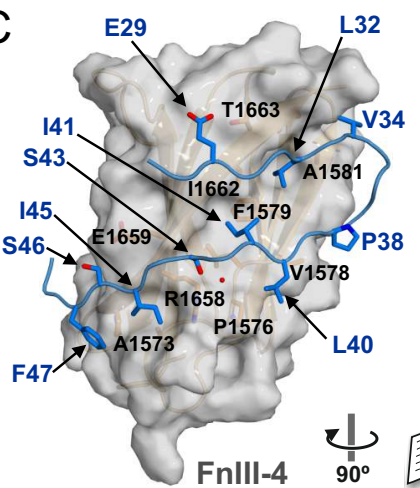

$E$

Mus musculus

Gallus gallus

Egretta garzetta

Pelodiscus sinensis

Alligator sinensis

Crotalus adamanteus

Latimeria chalumnae

$\begin{array}{lllllllllll}\text { Variable } & 1 & 2 & 3 & 4 & 5 & 6 & 7 & 8 & 9 & \text { Conserved }\end{array}$

$\mathrm{F}$

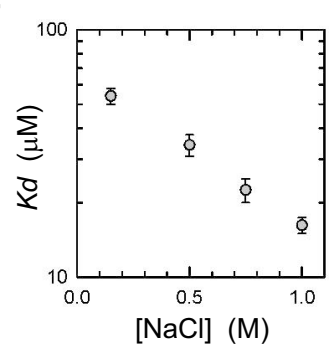

G
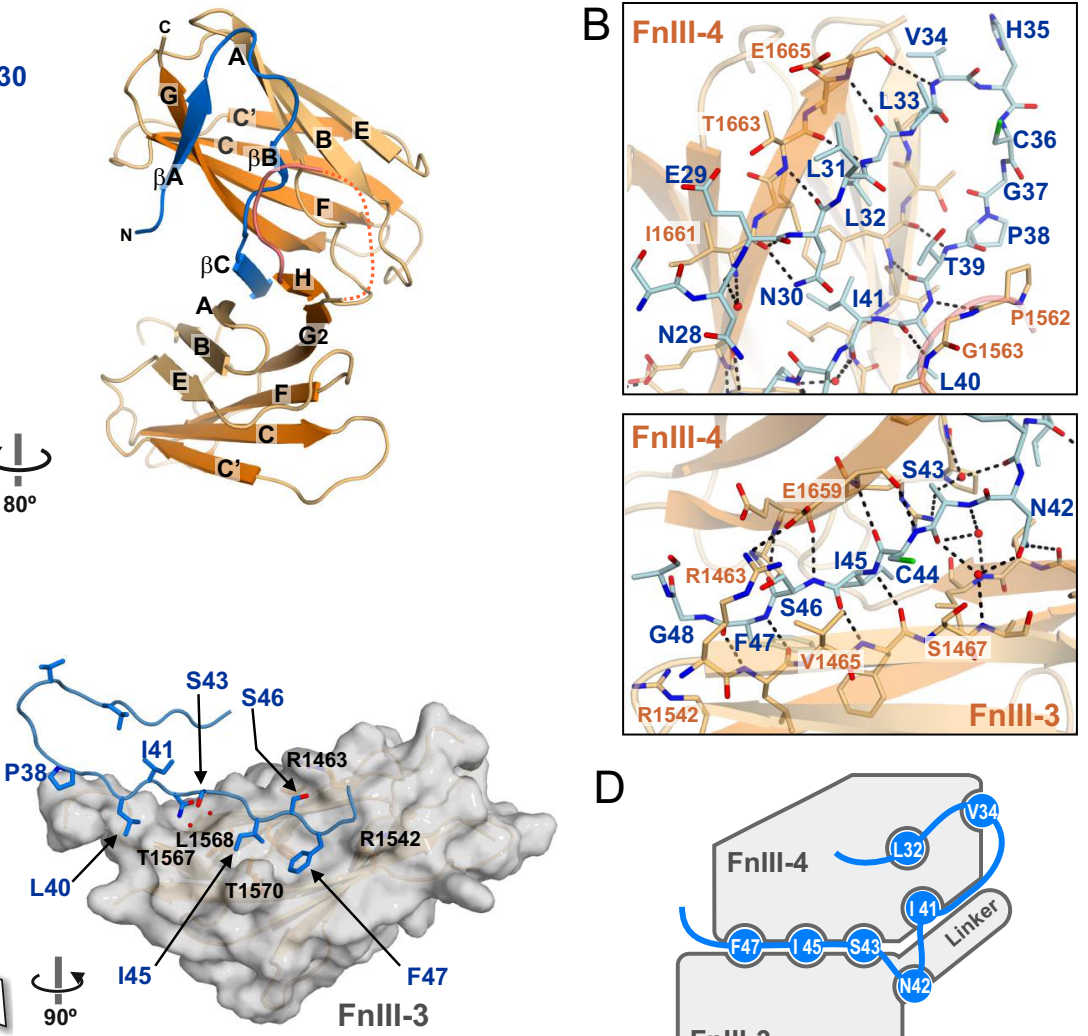

D

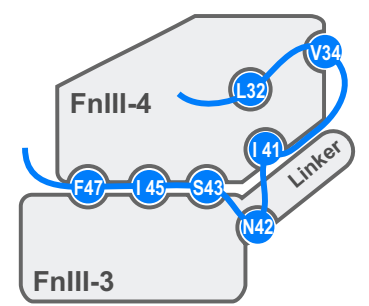

$\mathrm{H}$
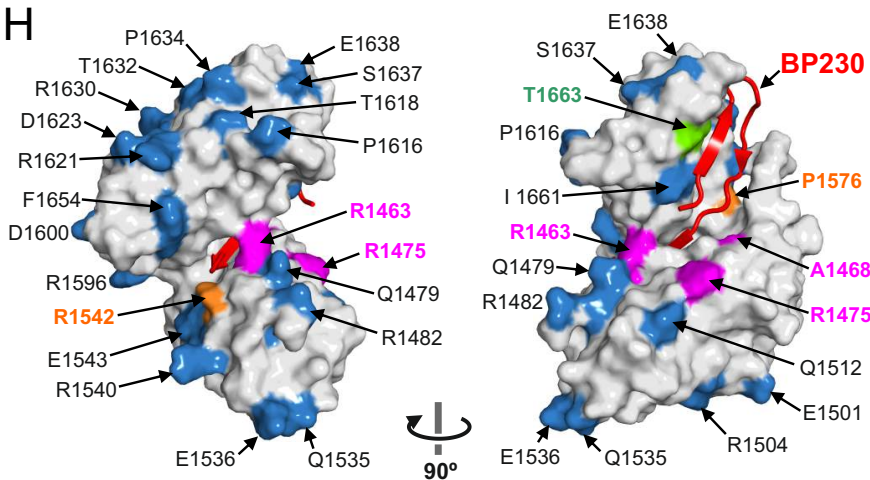

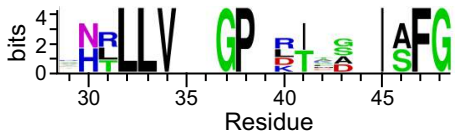

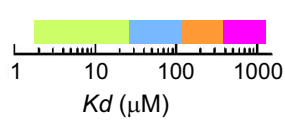


Fig 4

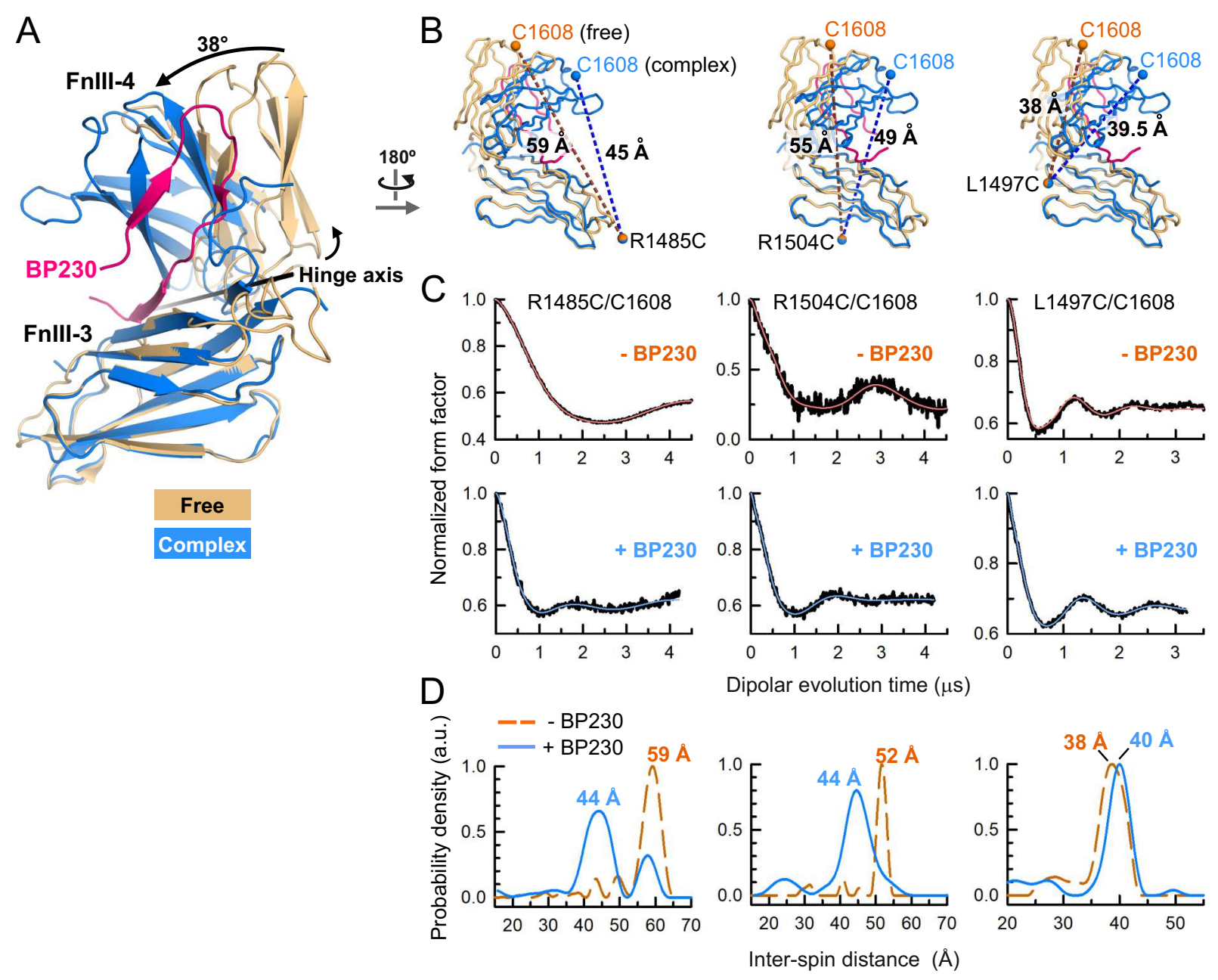


Fig 5
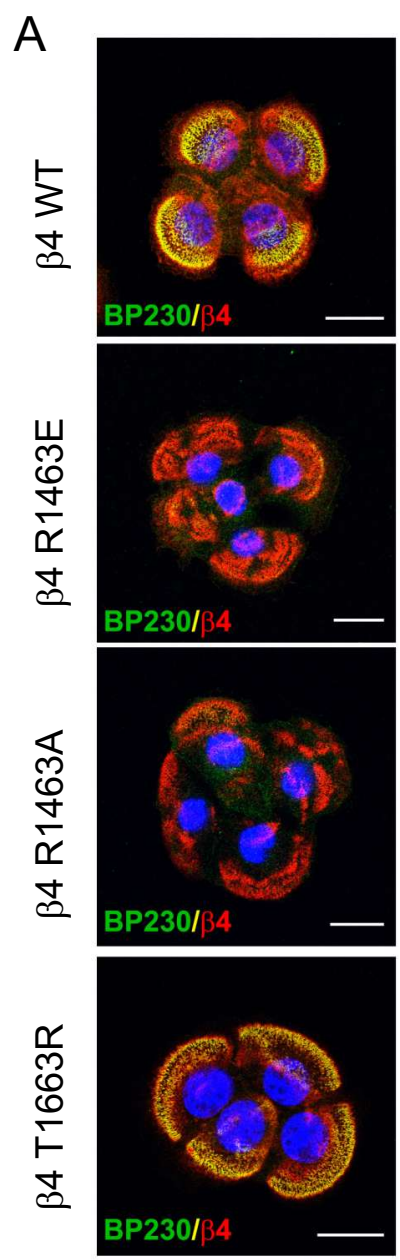
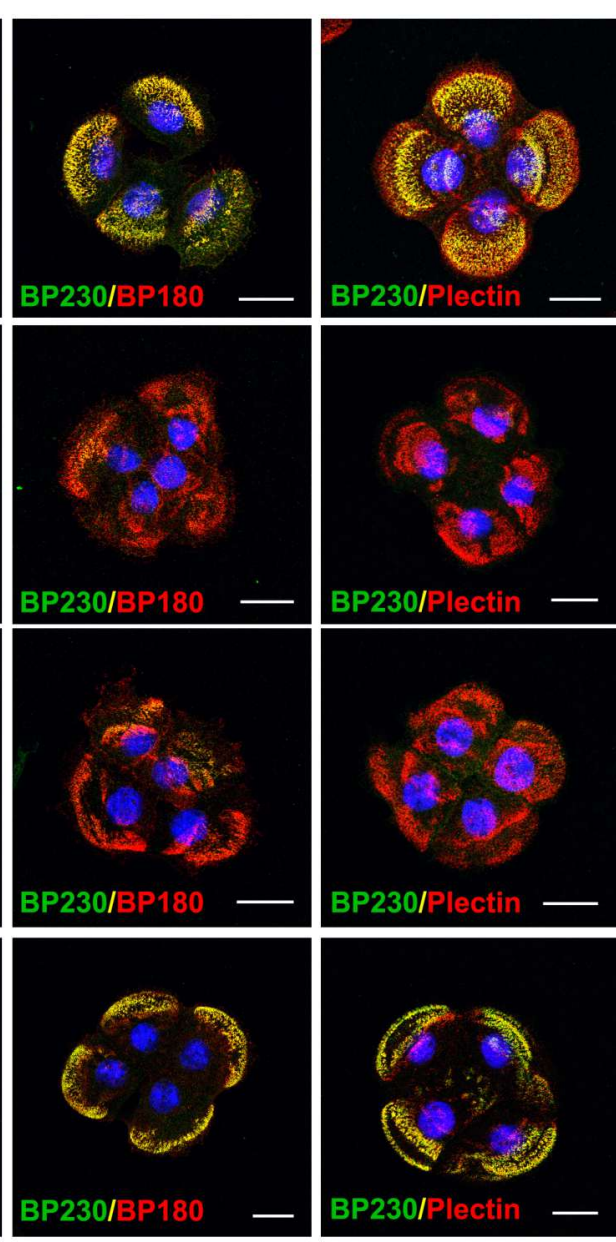

B
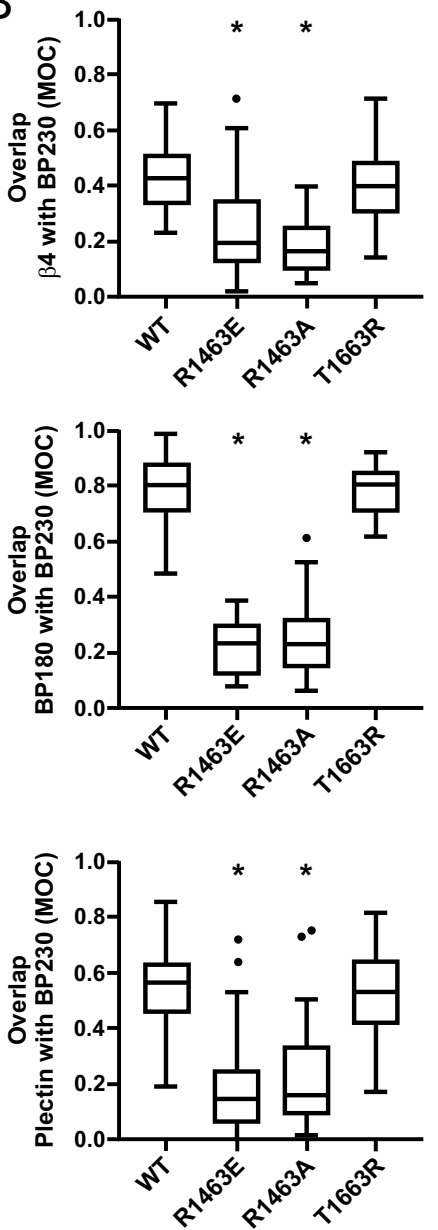
Fig 6

A

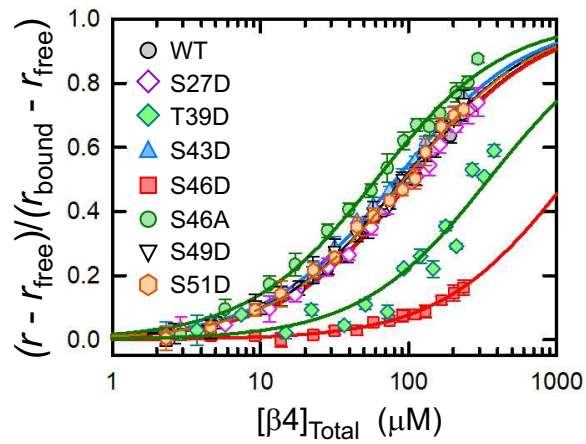

B

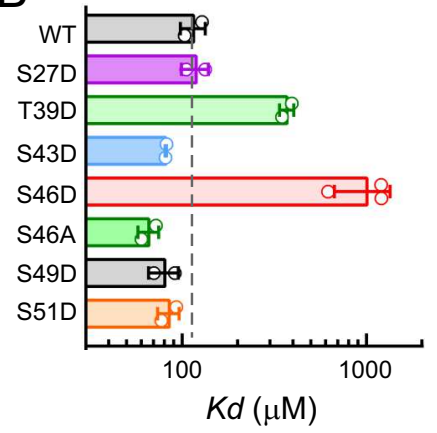

C
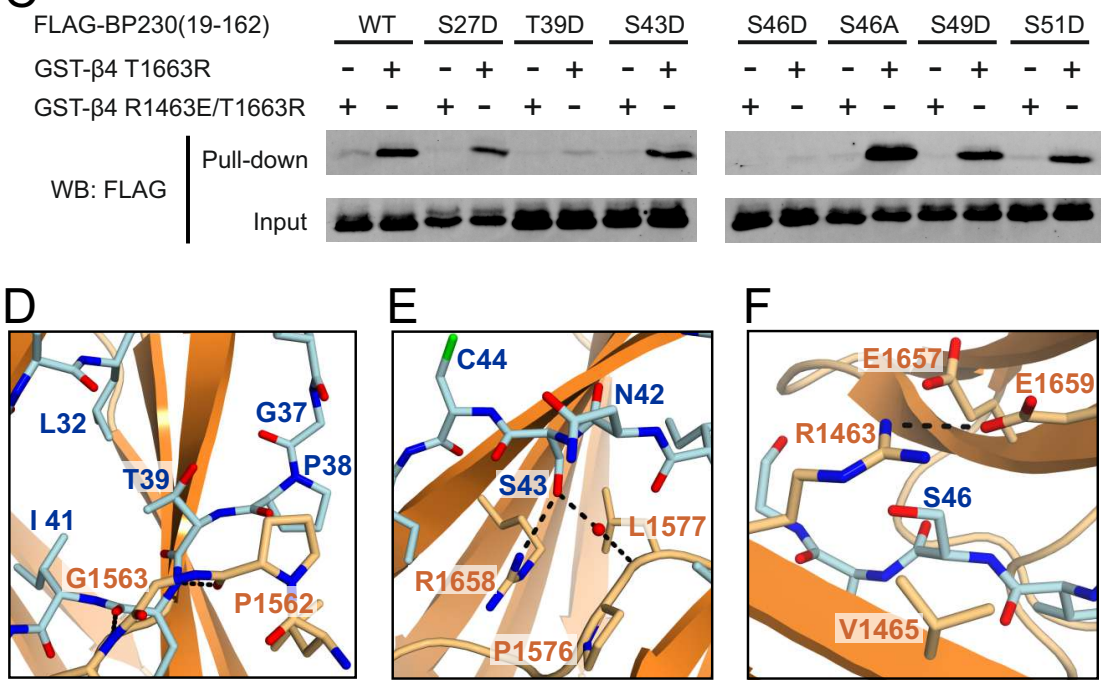
Fig 7

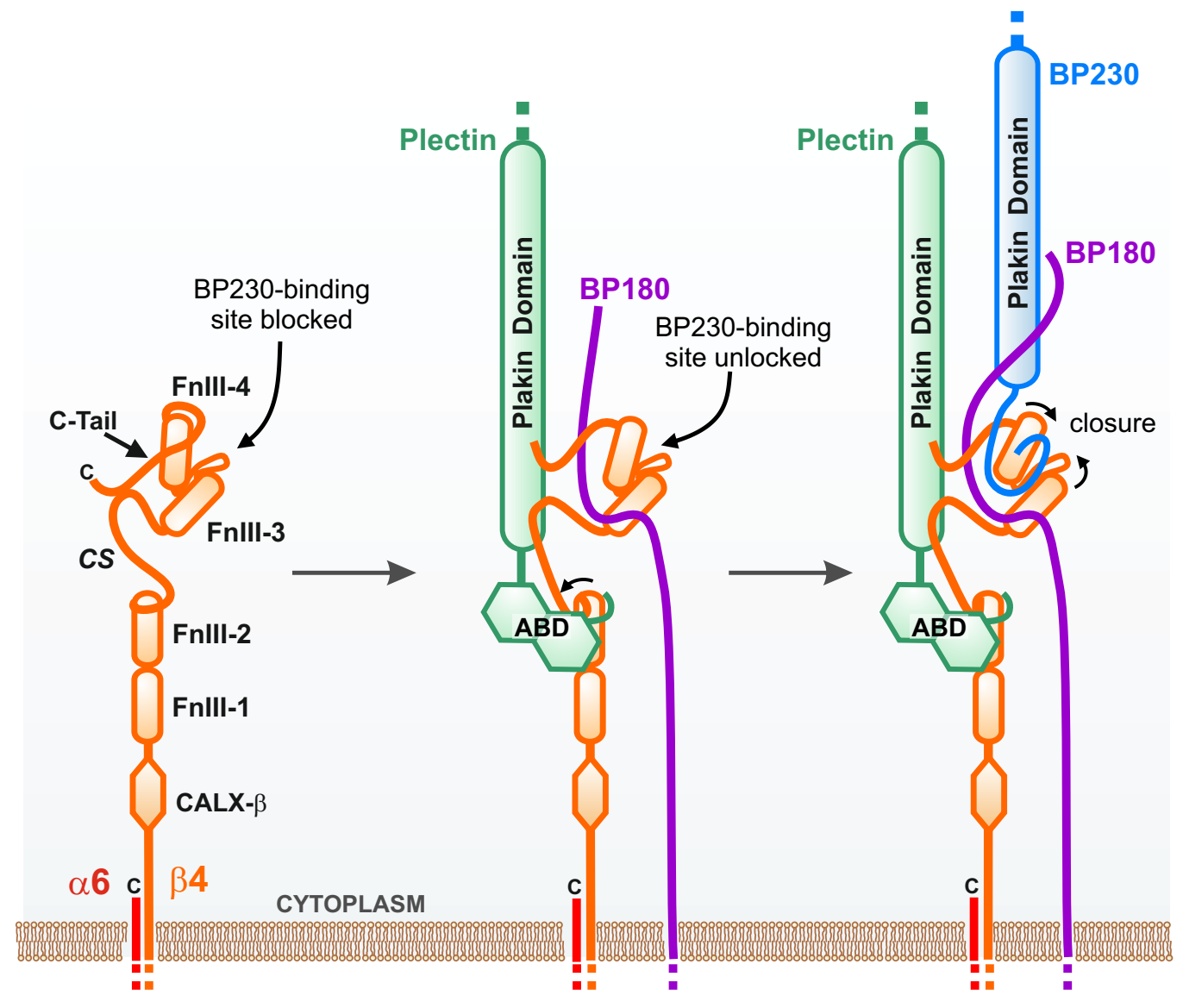



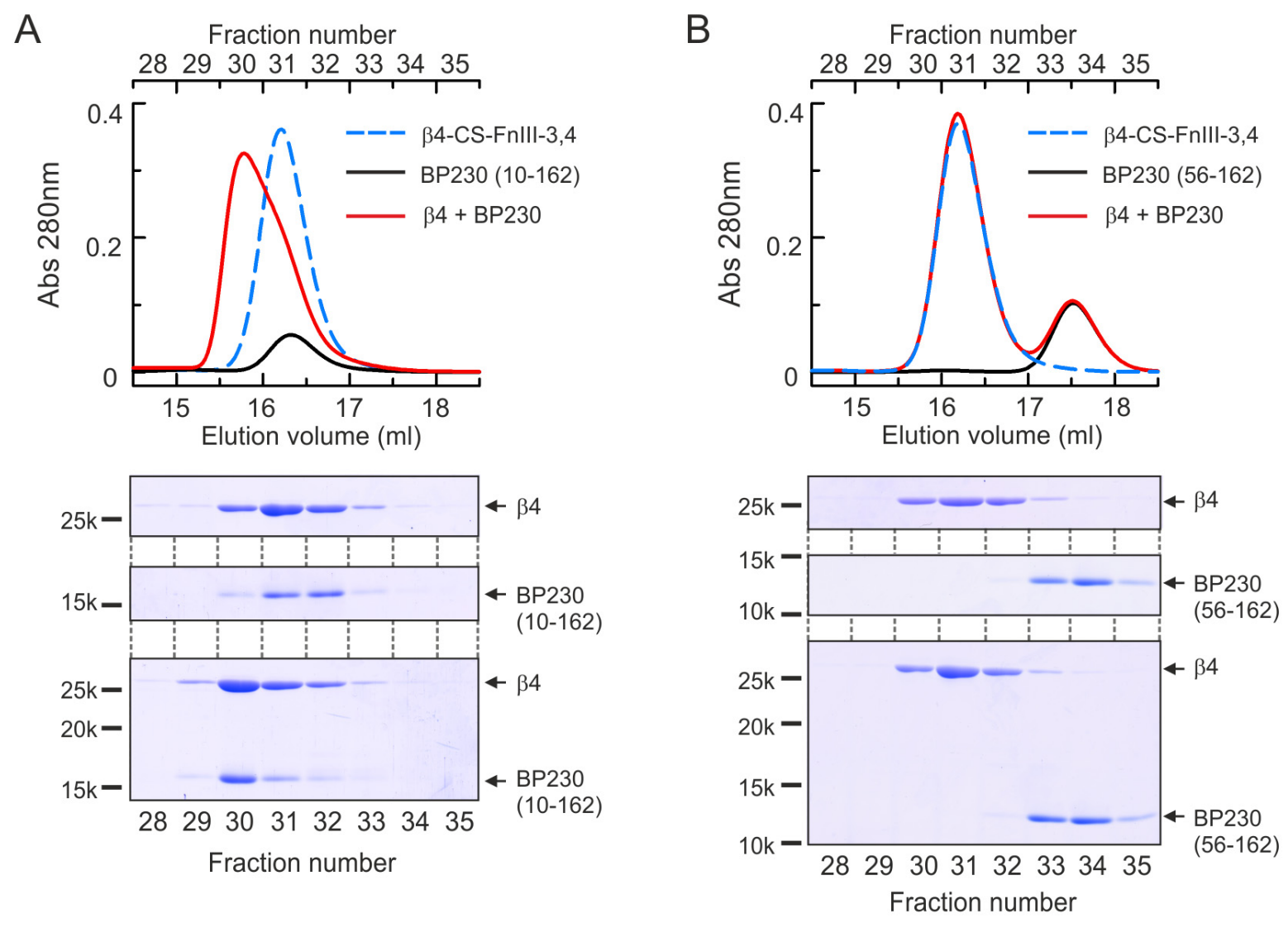

Figure. S1. Analysis by size exclusion chromatography of the interaction between $\beta 4-C S-F n I I I-3,4$ and the BP230 fragments 10-162 (A) and 56-162 (B), Related to Figure 1. Chromatograms of the isolated $\beta 4$ and BP230, and equimolar mixtures are shown. Samples were analyzed using a Superdex 200 10/300 GL column (GE Healthcare) equilibrated in $20 \mathrm{mM}$ Tris (pH 7.5), $150 \mathrm{mM} \mathrm{NaCl}, 0.5 \mathrm{mM}$ tris(2carboxyethyl)phosphine. The injection volume was $100 \mu \mathrm{l}$, the flow rate was $0.5 \mathrm{ml} / \mathrm{min}$, and fractions of $0.5 \mathrm{ml}$ were collected. Analyses of the fractions by SDS-PAGE are shown aligned in the bottom. 
A

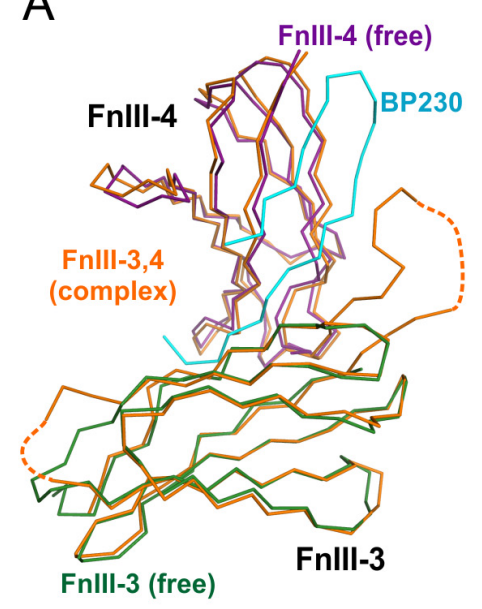

B

B

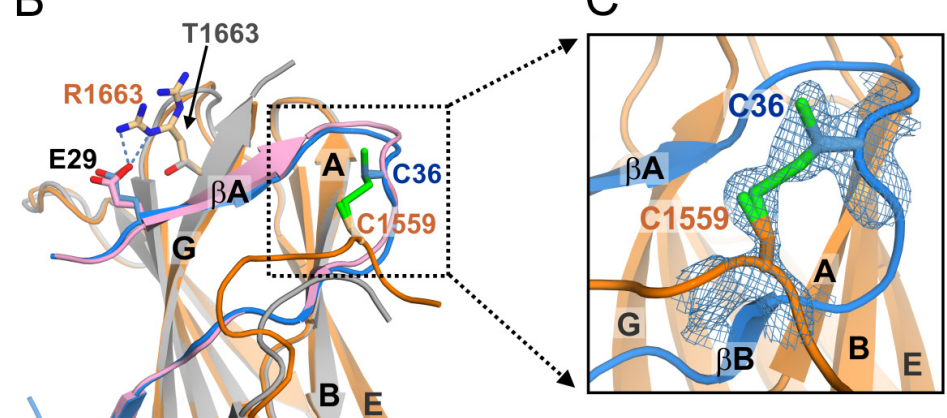

B4(T1663R) BP230

$\beta 4(W T) \quad B P 230$
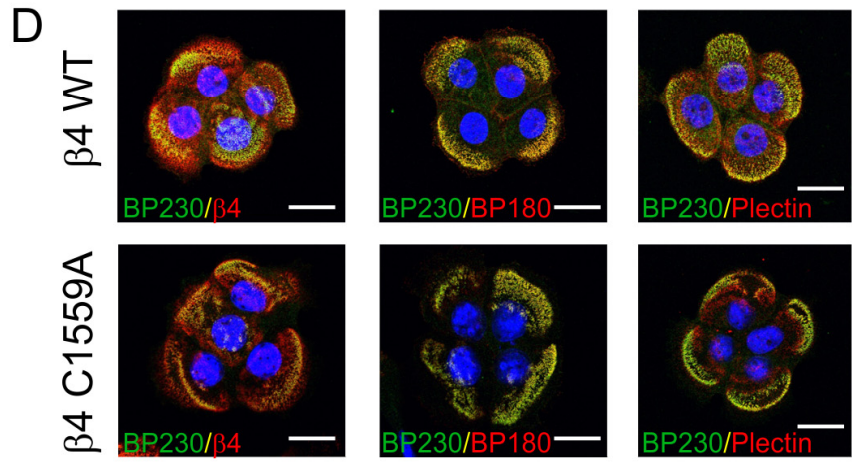

Figure S2. Details of the $\beta 4-B P 230$ complex and comparison of the WT and T1663R structures, Related to Figure 3. (A) Superimposition of the individual structures of the Fnlll-3 (dark green, PDB code 4WTW) and Fnlll-4 (dark violet, PDB 4WTX) domains onto the equivalent regions of the $\beta 4-B P 230$ structure ( $\beta 4$ orange, BP230 cyan). The structures are shown as Ca traces. (B) Ribbon representation of the $\beta 4(T 1663 R)-B P 230$ complex ( $\beta 4$ orange and BP230 blue) superimposed onto the $\beta 4(\mathrm{WT})-\mathrm{BP} 230$ complex ( $\beta 4$ grey and BP230 violet). For clarity, the Fnlll-3 is not shown. The side chains of $\beta 4$ T1663 (WT), the mutant R1663, and E29 of BP230 are shown as sticks. The salt bridge between $\beta 4-T 1663 R$ and BP230-E29 is shown as dashed lines. The side chains of $\beta 4-C 1559$ and BP230-C36, which were modeled as a partially formed disulfide bridge in the $\beta 4(\mathrm{~T} 1663 \mathrm{R})-\mathrm{BP} 230$ structure, are also shown. (C) Close-up view of the region around $\beta 4-\mathrm{C} 1559$ and BP230-C36 in the $\beta 4(\mathrm{~T} 1663 \mathrm{R})-\mathrm{BP} 230$ structure (dash square in A). A $2 m$ Fobs-DFcalc map (contoured at $1 \sigma$ ) is shown around the two cysteines. (D) Analysis of the role of the disulfide bond in the recruitment of BP230 in keratinocytes. Confocal microscopy images of PA-JEB keratinocytes expressing WT or C1559A $\beta 4$. Cells were stained pairwise with antibodies against BP230 and either $\beta 4, \mathrm{BP} 180$, or plectin. Nuclei were counterstained with DAPI (blue). Scale bar, $20 \mu \mathrm{m}$. When $\beta 4-C 1559 A$ was expressed in PA-JEB keratinocytes, it recruited endogenous BP230 to a similar extent as when WT $\beta 4$ was expressed, suggesting that C1559 is not required for the interaction with BP230. Thus, a physiological role cannot be assigned to the disulfide bridge, which might have formed during the crystallization. 

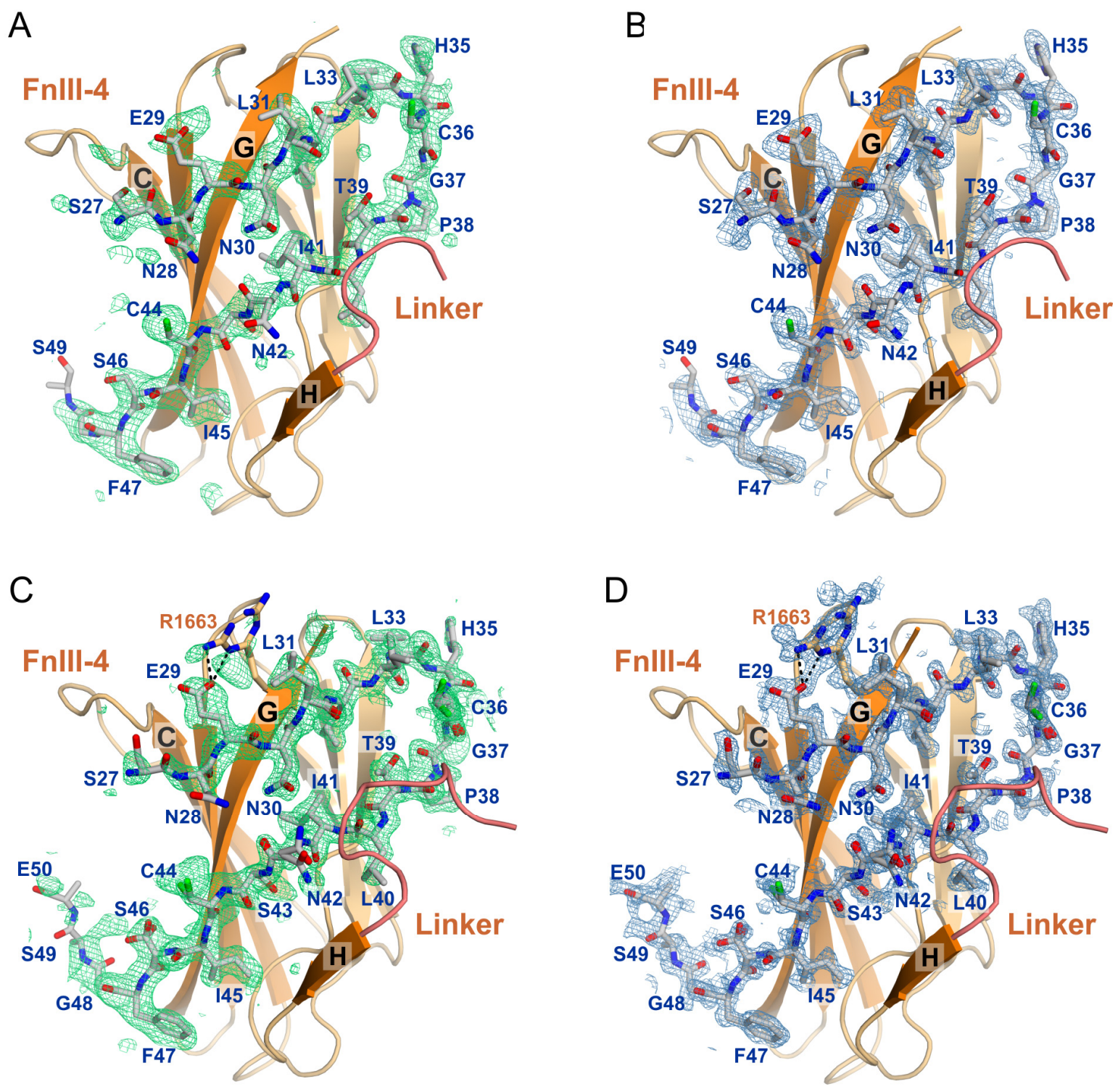

Figure S3. Electron density for BP230 in the structures of $\beta 4(W T)-B P 230$ (A, B) and $\beta 4(T 1663 R)$ BP230 (C, D), Related to Figure 3. (A, C) Composite omit difference maps ( $m F$ obs-DFcalc) contoured at $2.5 \sigma$ around BP230 (shown as sticks). Model bias was reduced by using a simulated annealing refinement protocol and by sequentially omitting parts of BP230 from the refinement. (B, D) Featureenhanced maps (FEM), 2mFobs-DFcalc, contoured at $1.5 \sigma$ around the BP230. For clarity, the FnIII-3 domain is not shown. 


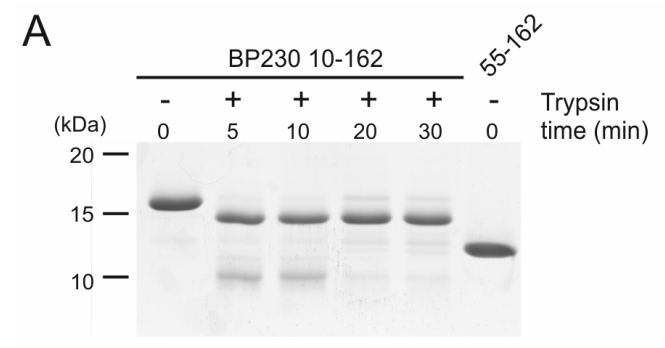

$E$
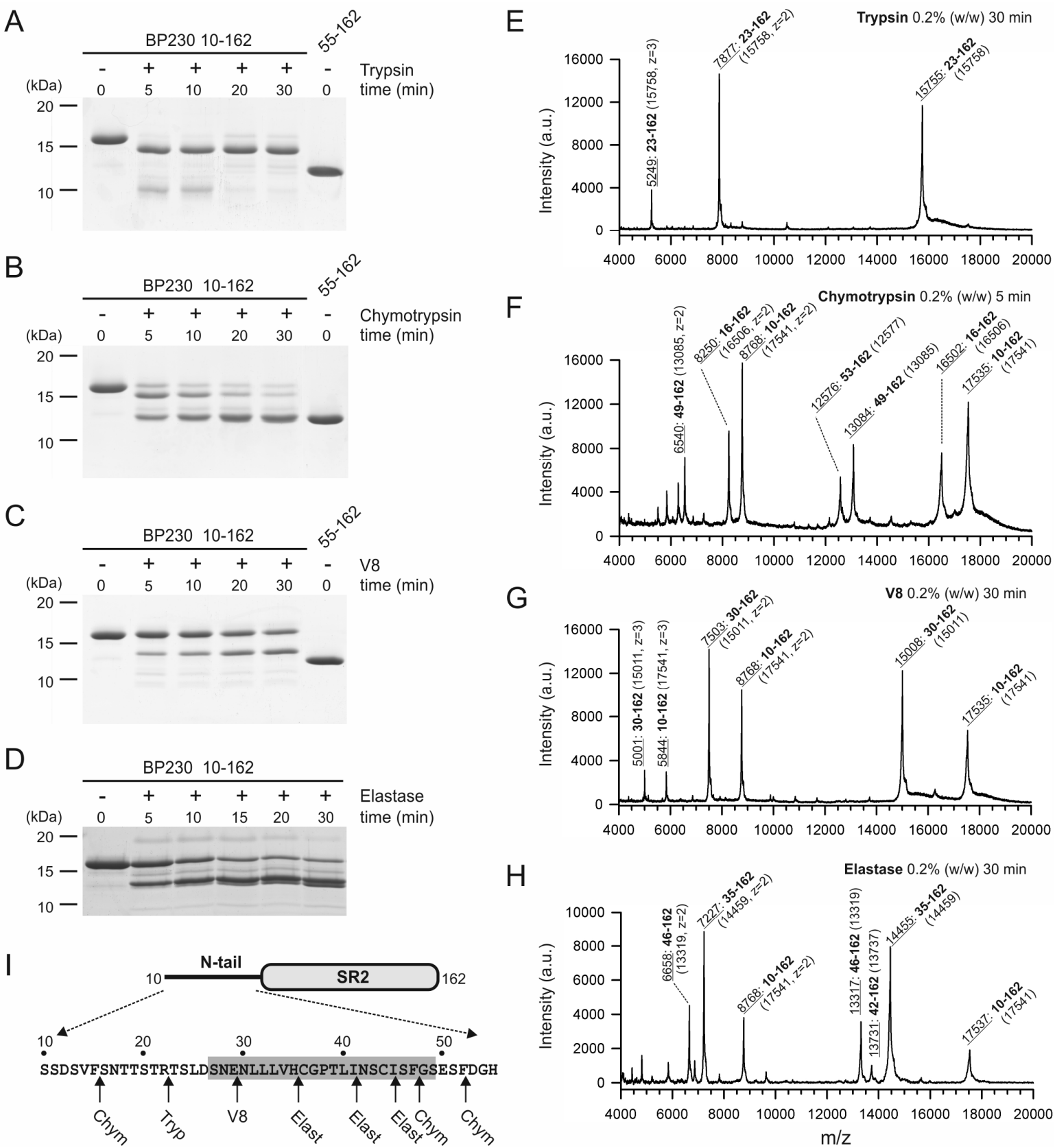

Figure S4. Limited proteolysis of BP230 in the absence of $\beta 4$, Related to Figure 3. (A-D) Analysis by SDS-PAGE (14\% acrylamide gels) of time course digestions of BP230 10-162 with $0.2 \%(\mathrm{w} / \mathrm{w})$ trypsin $(A)$, chymotrypsin (B), protease V8 (C), or elastase (D). In A-C the last lane corresponds to the SR2 domain (55-162). (E-H) analysis by MALDI-TOF mass spectrometry of the masses of the proteolytic fragments of BP230 10-162 as indicated. The experimental mass of each major peak is underlined. The corresponding region of BP230 is shown in bold and the calculated masses are in parenthesis. (I) Representation of the domain structure of BP230 10-162. All the digestion sites are located in the $\mathrm{N}$-tail as indicated under the sequence. The $\beta 4$-binding region is highlighted by a grey box. 
A

Homo sapiens
Gorilla gorilla
Pan troglodytes
Nomascus leucogenys
Cricetulus griseus
Mus musculus
Rattus norvegicus
Ornithorhynchus anatinus
Equus ferus
Pteropus vampyrus
Sus scrofa
Macaca mulatta
Chinchilla lanigera
Ursus maritimus
Canis lupus familiaris
Felis catus
Bos taurus
Callithrix jacchus
Dipodomys ordii
Tinamus guttatus
Meleagris gallopavo
Gallus gallus
Falco cherrug
Falco peregrinus
Egretta garzetta
Anas platyrhynchos
Ficedula albicollis
Haliaeetus leucocephalus
Aquila chrysaetos canadensis
Pelodiscus sinensis
Chelonia mydas
Anolis carolinensis
Alligator sinensis
Crotalus adamanteus
Ophiophagus hannah
Latimeria chalumnae

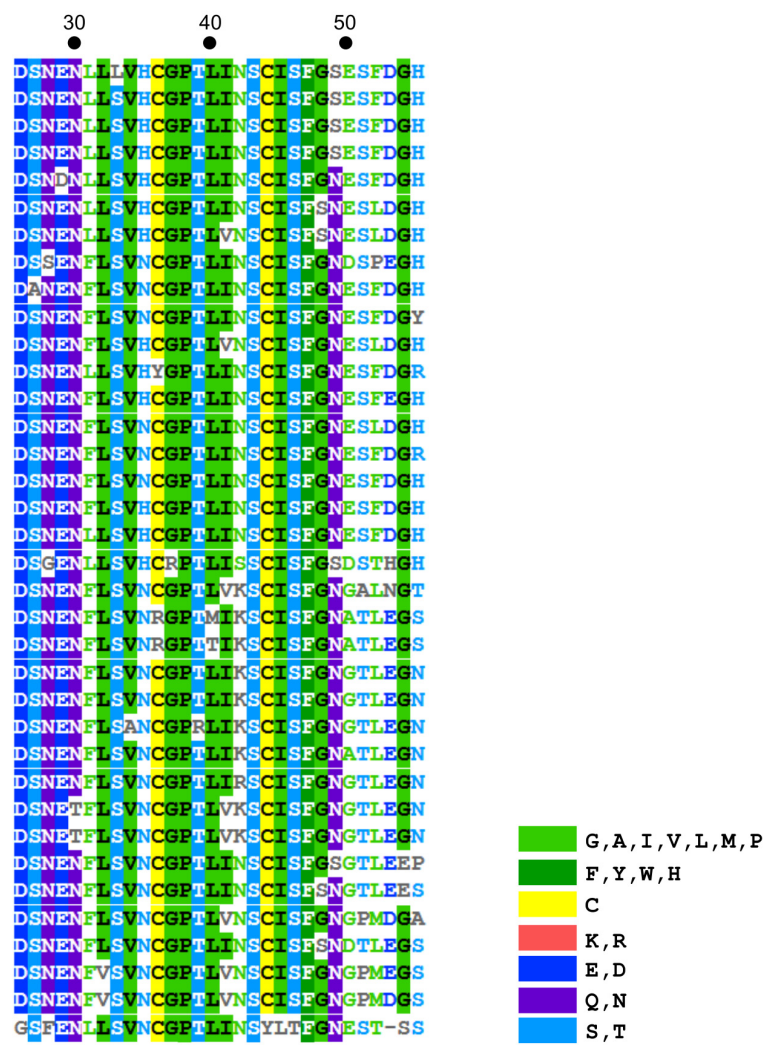

B

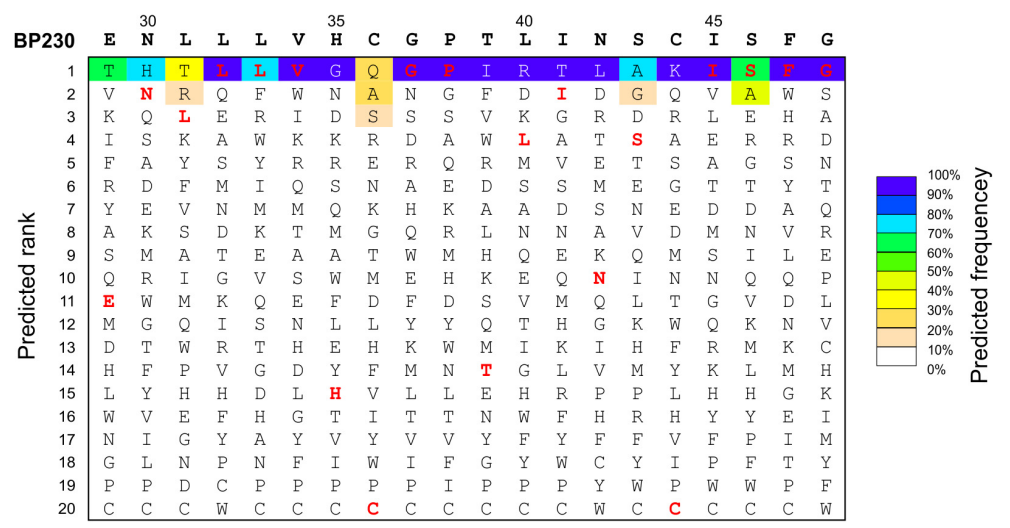

Figure S5. Analysis of the $\beta 4$-binding sequence of BP230, Related to Figure 3. (A) Alignment of the region 26-55 of human BP230 with the equivalent segments of orthologs from 35 species. Residues that match the consensus sequence $(80 \%)$ are highlighted by boxes colored according to the type of residue as indicated in the legend. (B) Prediction of the sequence tolerance in the $\beta 4$-binding site of BP230. Table of the amino acids ranked individually for each sequence position of the region 29-48 of BP230 according to the frequency with which they are predicted to be tolerated without significantly affecting the stability of BP230 and the binding interface. The effects of the amino acid substitutions were predicted with Rosetta (using a Boltzmann factor $k T=0.59$ ) using the structure of the WT $\beta 4-B P 230$ complex. The BP230 sequence is shown above. Wild type residues in the ranked list are shown in red. 

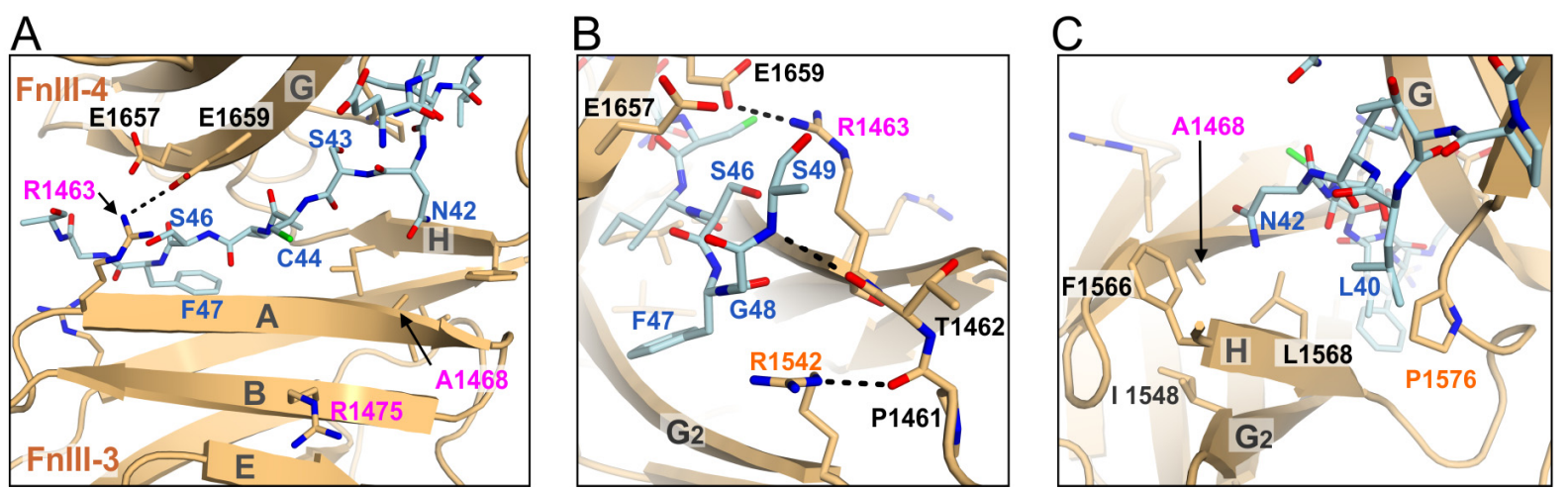

Figure S6. Structural interpretation of the mutagenesis data, Related to Figure 3. (A-C) Close ups of regions that include residues of $\beta 4$ that were shown in the mutagenesis analysis to be important for binding of BP230. 

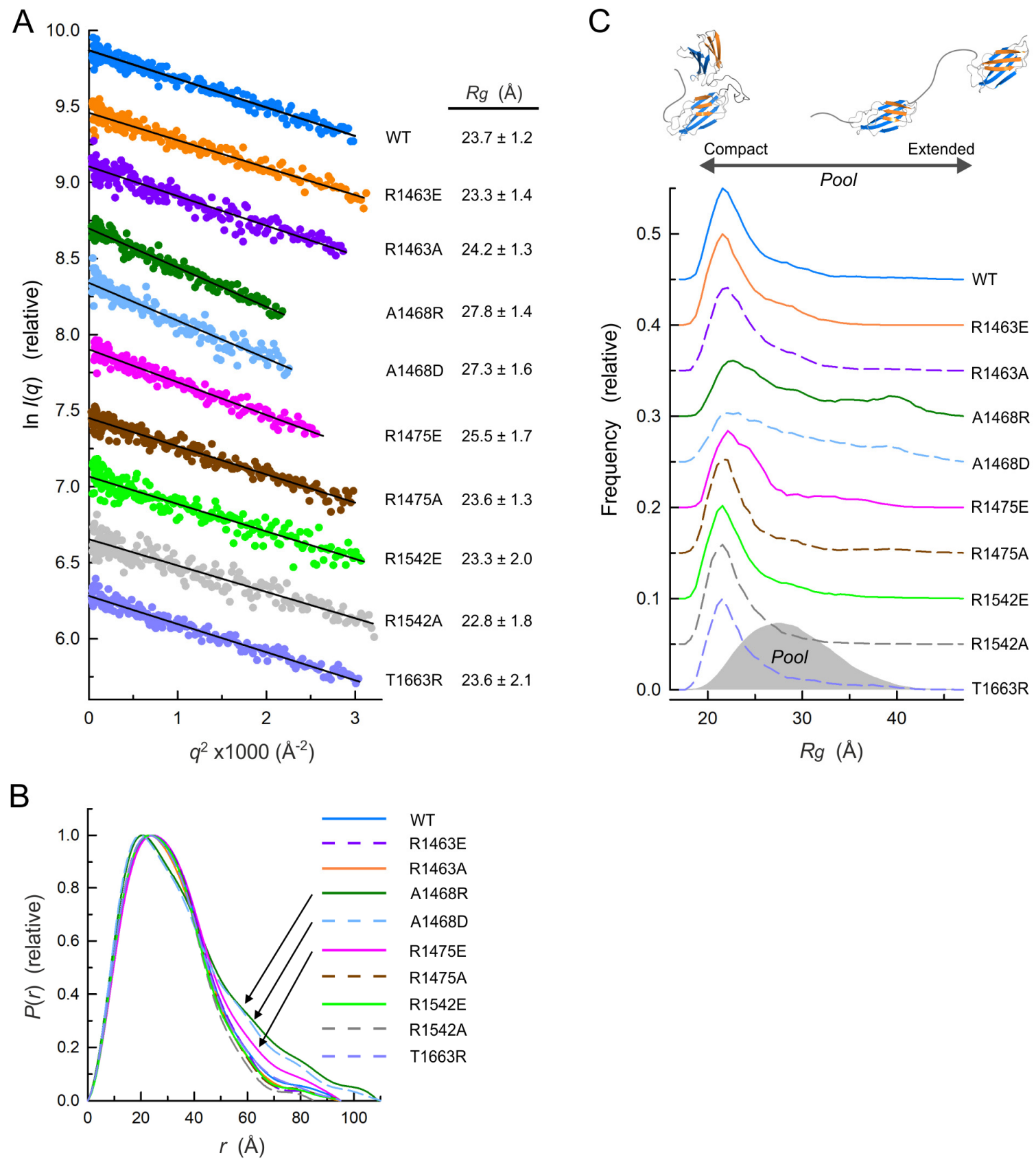

Figure S7. Analysis by SAXS of the effect of point mutations on the structure of the CS-FnIII-3,4 of $\beta 4$, Related to Figure 3. (A) Guinier plots of the SAXS data of $\beta 4-C S-F n l l l-3,4$ WT and mutants and the derived $R g$ values ( \pm STD). Lines are the Guinier fit. (B) $P(r)$ functions of $\beta 4$ WT and mutants. (C) EOM analysis of the flexibility in the WT and mutants of $\beta 4-C S-F n l l-3,4$. The frequency distributions of $R g$ in a pool of calculated models (grey area) and in the selected ensembles that fit the SAXS data of the WT and mutant proteins (lines) are shown. Plots in A and C are vertically displaced for representation. 

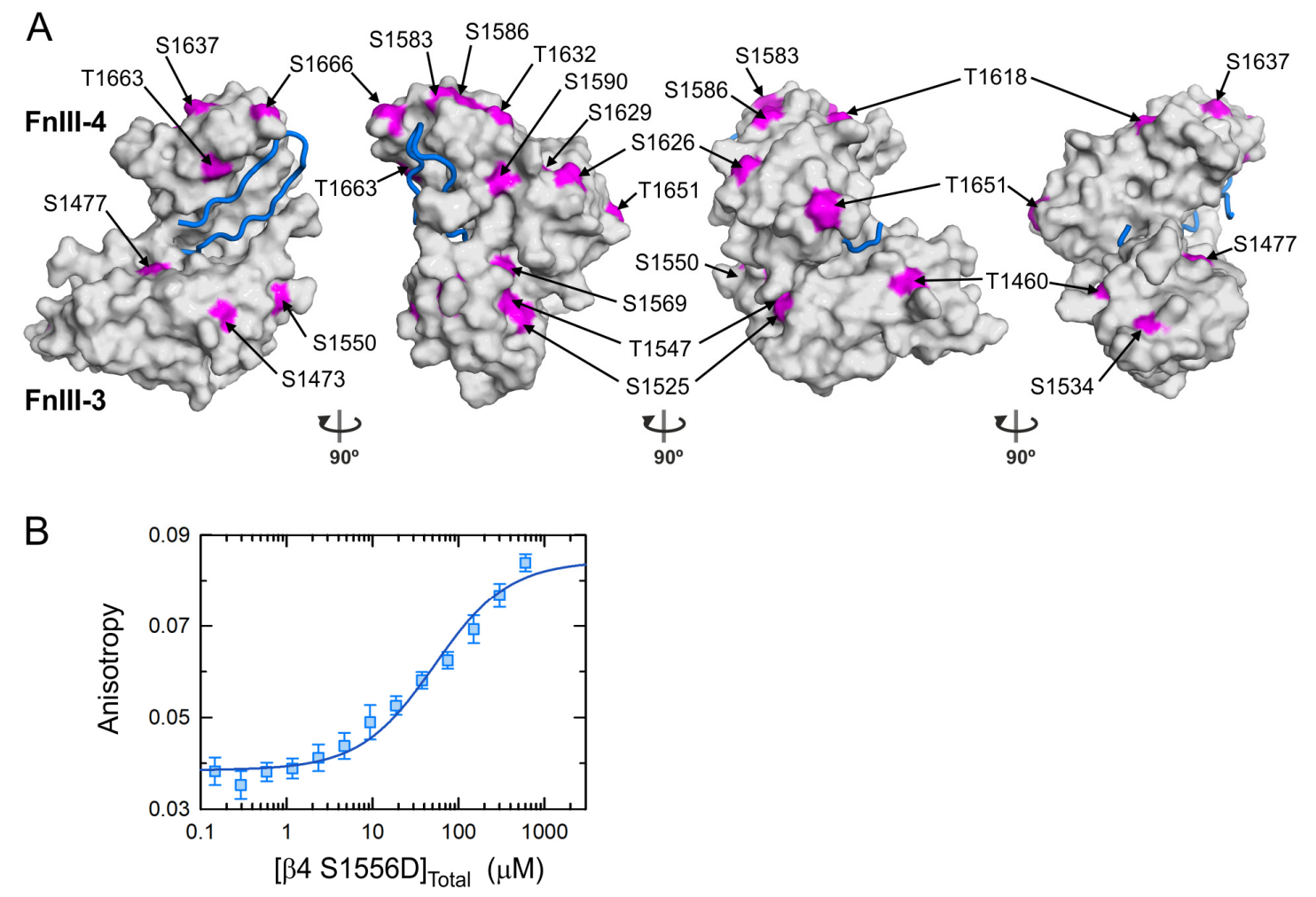

Figure S8. Putative phosphorylatable serine and threonine residues in the FnIII-3,4 of $\beta 4$, Related to Figure 6. (A) Surface representation of the Fnlll-3,4, with the backbone of BP230 shown as a blue wire. Ser/Thr residues in $\beta 4$ predicted to be potential phosphorylation sites by the Phosphonet server are shown in pink. (B) Binding of $\beta 4$ (1457-1666) carrying the phosphomimetic substitution S1556D to the fluorescein-labeled BP230 peptide 26-55 $(0.5 \mu \mathrm{M})$, measured by fluorescence anisotropy. The line represents the fit to the data, which corresponds to $K d=52 \pm 11 \mu \mathrm{M}$ (fitted value \pm standard error). 
Table S1. Affinity of $\beta 4-C S-F n I I I-3,4$ for BP230 fragments determined in individual experiments, Related to Figure 1D.

\begin{tabular}{|c|c|}
\hline BP230 fragment & $K d(\mu \mathrm{M})^{a}$ \\
\hline \multirow[t]{2}{*}{$10-162$} & $103 \pm 7$ \\
\hline & $128 \pm 12$ \\
\hline \multirow[t]{3}{*}{$19-162$} & $99 \pm 12$ \\
\hline & $107 \pm 18$ \\
\hline & $83 \pm 13$ \\
\hline \multirow[t]{2}{*}{ 28-162 } & $91 \pm 18$ \\
\hline & $94 \pm 11$ \\
\hline \multirow[t]{2}{*}{$32-162$} & $181 \pm 33$ \\
\hline & $255 \pm 40$ \\
\hline \multirow[t]{4}{*}{$37-162$} & $205 \pm 30$ \\
\hline & $138 \pm 11$ \\
\hline & $121 \pm 9$ \\
\hline & $110 \pm 8$ \\
\hline \multirow[t]{2}{*}{$41-162$} & $665 \pm 270$ \\
\hline & $513 \pm 86$ \\
\hline \multirow[t]{2}{*}{$46-162$} & $>2000^{b}$ \\
\hline & $>2000^{b}$ \\
\hline \multirow[t]{2}{*}{$56-162$} & $>2000^{b}$ \\
\hline & $>2000^{b}$ \\
\hline \multirow[t]{2}{*}{ 19-58/Ple SR2 } & $137 \pm 24$ \\
\hline & $116 \pm 17$ \\
\hline \multirow[t]{3}{*}{$26-55$} & $44 \pm 4$ \\
\hline & $50 \pm 6$ \\
\hline & $60 \pm 5$ \\
\hline \multirow[t]{4}{*}{$26-47$} & $200 \pm 27$ \\
\hline & $270 \pm 44$ \\
\hline & $224 \pm 42$ \\
\hline & $211 \pm 106$ \\
\hline \multirow[t]{2}{*}{$37-55$} & $148 \pm 7$ \\
\hline & $156 \pm 6$ \\
\hline
\end{tabular}

a , Data is presented as the estimated value \pm standard error of the fit.

b' Only a small saturation fraction could be achieved, which prevented the estimation of the $K d$ and the anisotropy of the bound state. Thus, a minimal value compatible with the data is shown. 
Table S2. Affinity of $\beta 4-C S-F n I I I-3,4$ mutants for BP230 (26-55) determined in individual experiments, Related to Figure 2B.

\begin{tabular}{|c|c|c|c|}
\hline$\beta 4$ & $K d(\mu \mathrm{M})^{a}$ & $\beta 4$ & $K d(\mu \mathrm{M})^{a}$ \\
\hline \multirow[t]{3}{*}{$\mathrm{WT}^{\mathrm{b}}$} & $44 \pm 4$ & R1540E & $82 \pm 7$ \\
\hline & $50 \pm 6$ & & $96 \pm 18$ \\
\hline & $60 \pm 5$ & R1540A & $62 \pm 8$ \\
\hline \multirow[t]{2}{*}{ R1463E } & $904 \pm 780$ & & $67 \pm 11$ \\
\hline & $650 \pm 184$ & R1542E & $499 \pm 129$ \\
\hline \multirow[t]{2}{*}{ R1463A } & $394 \pm 62$ & & $633 \pm 106$ \\
\hline & $232 \pm 60$ & R1542A & $163 \pm 23$ \\
\hline \multirow[t]{2}{*}{ A1468R } & $351 \pm 90$ & & $214 \pm 32$ \\
\hline & $293 \pm 85$ & E1541R/E1543R & $26 \pm 3$ \\
\hline \multirow[t]{2}{*}{ A1468D } & $1012 \pm 1318$ & & $30 \pm 4$ \\
\hline & $955 \pm 138$ & T1547R & $132 \pm 18$ \\
\hline \multirow[t]{2}{*}{ R1475E } & $578 \pm 583$ & & $109 \pm 13$ \\
\hline & $515 \pm 103$ & P1576R & $172 \pm 40$ \\
\hline \multirow[t]{2}{*}{$\mathrm{R} 1475 \mathrm{~A}$} & $443 \pm 174$ & & $152 \pm 18$ \\
\hline & $171 \pm 33$ & V1578R/T1580R & $57 \pm 10$ \\
\hline \multirow[t]{2}{*}{ Q1479R } & $59 \pm 9$ & R1595E/R1596E & $80 \pm 11$ \\
\hline & $76 \pm 14$ & & $99 \pm 15$ \\
\hline \multirow[t]{2}{*}{ E1480R } & $67 \pm 12$ & D1600R & $54 \pm 18$ \\
\hline & $88 \pm 14$ & & $75 \pm 12$ \\
\hline \multirow{2}{*}{ 1482RCER1485/ECRE } & $113 \pm 14$ & P1616R/T1618R & $70 \pm 10$ \\
\hline & $96 \pm 11$ & & $60 \pm 11$ \\
\hline \multirow[t]{2}{*}{ E1493R } & $100 \pm 33$ & R1621E/D1623R & $75 \pm 12$ \\
\hline & $99 \pm 13$ & & $99 \pm 14$ \\
\hline \multirow[t]{2}{*}{ N1498R } & $100 \pm 10$ & E1628R/R1630E & $92 \pm 15$ \\
\hline & $109 \pm 19$ & & $84 \pm 11$ \\
\hline \multirow[t]{2}{*}{ E1501R } & $54 \pm 7$ & T1632R/P1634R & $29 \pm 3$ \\
\hline & $70 \pm 7$ & & $37 \pm 6$ \\
\hline \multirow[t]{2}{*}{ R1504E } & $99 \pm 12$ & S1637R/E1638R & $74 \pm 23$ \\
\hline & $61 \pm 2$ & F1654R & $20 \pm 3$ \\
\hline \multirow[t]{2}{*}{ Q1512R } & $53 \pm 5$ & & $30 \pm 6$ \\
\hline & $47 \pm 8$ & & $33 \pm 6$ \\
\hline \multirow[t]{2}{*}{ Q1535R/E1536R } & $407 \pm 158$ & I1661R/T1663R & $7 \pm 1$ \\
\hline & $518 \pm 151$ & I1661R & $56 \pm 8$ \\
\hline \multirow[t]{2}{*}{ Q1535R } & $71 \pm 10$ & & $65 \pm 8$ \\
\hline & $85 \pm 13$ & & $57 \pm 12$ \\
\hline \multirow[t]{2}{*}{ Q1535A } & $92 \pm 12$ & T1663R & $3.7 \pm 0.7$ \\
\hline & $63 \pm 12$ & & $2.0 \pm 0.3$ \\
\hline \multirow[t]{2}{*}{ E1536R } & $61 \pm 8$ & & $2.4 \pm 0.5$ \\
\hline & $87 \pm 13$ & & $5.3 \pm 1.4$ \\
\hline \multirow[t]{2}{*}{ E1536A } & $68 \pm 10$ & & $5.2 \pm 1.5$ \\
\hline & $78 \pm 18$ & T1663D & $423 \pm 93$ \\
\hline \multirow[t]{2}{*}{ R1540E/R1542E } & $756 \pm 291$ & & $326 \pm 77$ \\
\hline & $533 \pm 122$ & & \\
\hline
\end{tabular}


Table S3. SAXS data collection and derived parameters of $\beta 4-F n I I I-3,4$ proteins, Related to Figure 3.

\begin{tabular}{|c|c|c|c|c|c|c|c|c|c|c|}
\hline & WT & R1463E & R1463A & A1468R & A1468D & R1475E & R1475A & R1542E & R1542A & T1663R \\
\hline \multicolumn{11}{|l|}{ Data collection } \\
\hline Concentration range $\left(\mathrm{mg} \mathrm{ml}^{-1}\right)$ & $0.9-14.9$ & $1.4-5.5$ & $0.9-7.1$ & $1.5-11.9$ & $0.9-14.9$ & $1.2-9.2$ & $1.3-10.5$ & $0.9-14.8$ & $0.9-14.5$ & $1.5-11.7$ \\
\hline Exposure time (sec) & $30 \times 0.05$ & $30 \times 0.05$ & $30 \times 0.05$ & $30 \times 0.05$ & $30 \times 0.05$ & $30 \times 0.05$ & $30 \times 0.05$ & $30 \times 0.05$ & $30 \times 0.05$ & $30 \times 0.05$ \\
\hline \multicolumn{11}{|l|}{ Structural parameters } \\
\hline \multicolumn{11}{|l|}{ Guinier analysis } \\
\hline$q R_{\mathrm{g}}$ range & $0.15-1.30$ & $0.15-1.30$ & $0.18-1.30$ & $0.16-1.30$ & $0.16-1.30$ & $0.20-1.29$ & $0.13-1.30$ & $0.15-1.29$ & $0.15-1.30$ & $0.13-1.30$ \\
\hline$I(0) / \mathrm{c}\left(10^{-2} \mathrm{~cm}^{2} \mathrm{mg}^{-1}\right)^{\mathrm{a}}$ & 1.60 & 1.58 & 1.64 & 1.62 & 1.53 & 1.89 & 1.43 & 1.54 & 1.52 & 1.55 \\
\hline$R_{\mathrm{g}}(\AA)$ & $23.7 \pm 1.2$ & $23.3 \pm 1.4$ & $24.2 \pm 1.3$ & $27.8 \pm 1.4$ & $27.3 \pm 1.6$ & $25.5 \pm 1.7$ & $23.6 \pm 1.3$ & $23.3 \pm 2.0$ & $22.8 \pm 1.8$ & $23.6 \pm 2.1$ \\
\hline \multicolumn{11}{|l|}{$P(r)$ analysis } \\
\hline$I(0) / \mathrm{c}\left(10^{-2} \mathrm{~cm}^{2} \mathrm{mg}^{-1}\right)^{a}$ & 1.62 & 1.59 & 1.64 & 1.64 & 1.53 & 1.90 & 1.44 & 1.55 & 1.51 & 1.57 \\
\hline$R_{\mathrm{g}}(\AA)$ & 25.2 & 24.4 & 24.4 & 29.7 & 28.7 & 26.6 & 24.3 & 24.2 & 23.2 & 24.9 \\
\hline$D_{\max }(\AA)$ & 95 & 95 & 95 & 109 & 110 & 95 & 95 & 95 & 85 & 95 \\
\hline Porod volume, $\mathrm{Vp}\left(\AA^{3}\right)$ & 36900 & 35400 & 36100 & 35300 & 34200 & 42300 & 35400 & 35100 & 34100 & 35300 \\
\hline $\begin{array}{l}\text { Molecular mass }(\mathrm{kDa}) \text { [from } \\
(\mathrm{Vp} / 1.5) \text { ] }\end{array}$ & 24.6 & 23.6 & 24.1 & 23.5 & 22.8 & 28.2 & 23.6 & 23.4 & 22.7 & 23.5 \\
\hline $\begin{array}{l}\text { Monomeric mass from } \\
\text { sequence }(\mathrm{kDa})\end{array}$ & 25.6 & 25.6 & 25.6 & 25.6 & 25.6 & 25.6 & 25.6 & 25.6 & 25.6 & 25.6 \\
\hline SASBDB code & SASDDE8 & SASDDF8 & SASDDG8 & SASDDH8 & SASDDJ8 & SASDDK8 & SASDDL8 & SASDDM8 & SASDDN8 & SASDDP8 \\
\hline
\end{tabular}

a, Absolute intensities determined using water as a secondary standard. 
Table S4. Affinity of $\beta 4-C S-F n l l l-3,4$ for point mutants of BP230 (10-162) determined in individual experiments, Related to Figure 6B.

\begin{tabular}{cc}
\hline BP230 (10-162) & $K d(\mu \mathrm{M})^{\text {a }}$ \\
\hline WT & $103 \pm 7$ \\
& $128 \pm 12$ \\
S27D & $105 \pm 8$ \\
& $133 \pm 9$ \\
T39D & $347 \pm 232$ \\
& $393 \pm 213$ \\
S43D & $82 \pm 6$ \\
& $81 \pm 9$ \\
S46D & $1201 \pm 875$ \\
& $617 \pm 396$ \\
& $1197 \pm 1434$ \\
S46A & $72 \pm 6$ \\
& $60 \pm 7$ \\
S49D & $70 \pm 6$ \\
& $91 \pm 12$ \\
S51D & $93 \pm 12$ \\
& $77 \pm 9$ \\
\hline
\end{tabular}


Table S5. Primers used to subclone regions of $\beta 4$ and BP230 in expression vectors, Related to STAR Methods.

\begin{tabular}{|c|c|}
\hline \begin{tabular}{|l|} 
Primer name \\
\end{tabular} & Sequence (5' - 3') \\
\hline$\beta 4-1436$ NdeI Forward & $\begin{array}{l}\text { TGAGAATTCCATATGCTGCCGAGGGACTACTCCACC } \\
\qquad \begin{array}{llllllll}\text { NdeI } & \text { L } & \text { P } & \text { R } & \text { D } & \text { Y } & \text { S } & \text { T }\end{array}\end{array}$ \\
\hline$\beta 4-1666$ Stop-BamHI Reverse & \begin{tabular}{|l} 
GCCGAATTCGGATCCCTAGGACTCTATGGTGATGATGC \\
$\begin{array}{ccccccc}\text { BamHI } & \text { S } & \text { E } & \text { I } & \text { T } & \text { I } & \text { I }\end{array}$
\end{tabular} \\
\hline BP230-01 NdeI Forward & $\begin{array}{l}\text { TGAGAATTCCATATGCACAGTAGTAGTTATAGTTAC } \\
\qquad \begin{array}{llllllll}\text { NdeI } & \text { H } & \text { S } & \text { S } & \text { S } & Y & \text { S } & Y \\
\end{array}\end{array}$ \\
\hline BP230-10 NdeI Forward & \begin{tabular}{|l} 
TGAGAATTCCATATGAGCAGTGATTCTGTGTTTAG \\
$\qquad \begin{array}{llllll}\text { NdeI } & \text { S S D S V F }\end{array}$
\end{tabular} \\
\hline BP230-19 EcoRI-NdeI Forward & 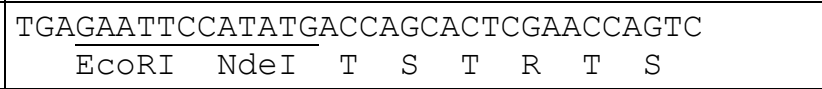 \\
\hline BP230-28 NdeI Forward & $\begin{array}{l}\text { TGAGAATTCCATATGAATGAAAATCTTCTCTTGGTTC } \\
\qquad \begin{array}{cccccccc}\text { NdeI } & N & \text { E } & \text { N } & \text { L } & \text { L } & \text { L } & \text { V }\end{array}\end{array}$ \\
\hline BP230-32 NdeI Forward & \begin{tabular}{|l} 
TGAGAATTCCATATGCTCTTGGTTCATTGTGGTCCAAC \\
$\qquad \begin{array}{llllllll}\text { NdeI } & \text { L } & \text { L } & \text { V } & \text { H } & \text { C } & \text { G } & \text { P }\end{array}$
\end{tabular} \\
\hline BP230-37 NdeI Forward & 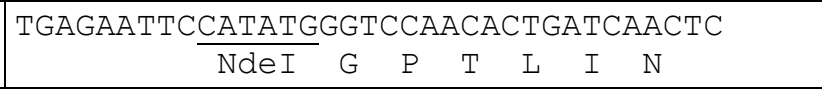 \\
\hline BP230-41 NdeI Forward & $\begin{array}{l}\text { TGAGAATTCCATATGATCAACTCTTGCATTAGCTTCGGC } \\
\qquad \begin{array}{lllllllll}\text { NdeI } & \text { I } & \text { N } & \text { S } & \text { C } & \text { I } & \text { S } & \text { F } & \text { G }\end{array}\end{array}$ \\
\hline BP230-46 NdeI Forward & $\begin{array}{l}\text { TGAGAATTCCATATGAGCTTCGGCAGTGAATCC } \\
\qquad \begin{array}{lllllll}\text { NdeI } & S & \text { F } & \text { G } & \text { S } & \text { E } & \text { S }\end{array}\end{array}$ \\
\hline BP230-56 NdeI Forward & $\begin{array}{l}\text { TGAGAATTCCATATGAGGTTAGAAATGTTGCAACAG } \\
\qquad \begin{array}{llllllll}\text { NdeI } & \text { R } & \text { L } & \text { E } & \text { M } & \text { L } & Q & Q \\
\end{array}\end{array}$ \\
\hline BP230-162 Stop-BamHI Reverse & 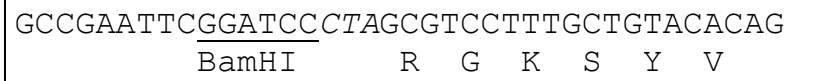 \\
\hline BP230-58/PLE-420 Forward & $\begin{array}{l}\text { CTTTGATGGACACAGGTTAGAATGTCTTCAGCGCATCGTG } \\
\begin{array}{ccccccccccccc}\text { F } & \text { D } & \text { G } & \text { H } & \text { R } & \text { L } & \text { E } & \text { C } & \text { L } & \text { Q } & \text { R } & \text { I } & \text { V }\end{array}\end{array}$ \\
\hline BP230-58/PLE-420 Reverse & $\begin{array}{l}\text { CACGATGCGCTGAAGACATTCTAACCTGTGTCCATCAAAG } \\
\begin{array}{ccccccccccccc}\mathrm{V} & \mathrm{I} & \mathrm{R} & \mathrm{Q} & \mathrm{L} & \mathrm{C} & \mathrm{E} & \mathrm{L} & \mathrm{R} & \mathrm{H} & \mathrm{G} & \mathrm{D} & \mathrm{F} \\
\end{array}\end{array}$ \\
\hline BP230-162 Stop-NotI Reverse & 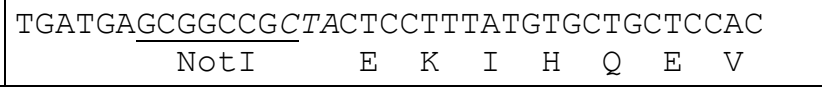 \\
\hline
\end{tabular}


Table S6. Primers used for site directed mutagenesis of $\beta 4$, Related to STAR Methods.

\begin{tabular}{|c|c|}
\hline Primer name & Sequence (5' - 3') \\
\hline$\beta 4$ R1463E Forward & GTGCCCGACACGCCCACCGAGCTGGTGTTCTC \\
\hline$\beta 4$ R1463E Reverse & GAGAACACCAGCTCGGTGGGCGTGTCGGGCAC \\
\hline$\beta 4$ R1463A Forward & CCGACACGCCCACCGCCCTGGTGTTCTC \\
\hline$\beta 4$ R1463A Reverse & GAGAACACCAGGGCGGTGGGCGTGTCGG \\
\hline$\beta 4$ A1468R Forward & СTGGTGTTCTCTCGCCTGGGGCCCACATCTC \\
\hline$\beta 4$ A1468R Reverse & GAGATGTGGGCCCCAGGCGAGAGAACACCAG \\
\hline$\beta 4$ A1468D Forward & СCTGGTGTTCTCTGACCTGGGGCCCAC \\
\hline$\beta 4$ A1468D Reverse & GTGGGCCCCAGGTCAGAGAACACCAGG \\
\hline$\beta 4$ R1475E Forward & CTGGGGCCCACATCTCTCGAAGTGAGCTGGC \\
\hline$\beta 4$ R1475E Reverse & GCCAGCTCACTTCGAGAGATGTGGGCCCCAG \\
\hline$\beta 4$ R1475A Forward & GGGCCCACATCTCTCGCAGTGAGCTGGCAG \\
\hline$\beta 4$ R1475A Reverse & CTGCCAGCTCACTGCGAGAGATGTGGGCCC \\
\hline$\beta 4$ Q1479R Forward & CAGAGTGAGCTGGCGGGAGCCGCGGTGCG \\
\hline$\beta 4$ Q1479R Reverse & CGCACCGCGGCTCCCGCCAGCTCACTCTG \\
\hline$\beta 4$ E1480R Forward & CAGAGTGAGCTGGCAGCGGCCGCGGTGCGAGC \\
\hline$\beta 4$ E1480R Reverse & GCTCGCACCGCGGCCGCTGCCAGCTCACTCTG \\
\hline$\beta 4$ R1482E-R1485E Forward & CTGGCAGGAGCCGGAGTGCGAGGAGCCGCTGCAG \\
\hline$\beta 4$ R1482E-R1485E Reverse & СTGCAGCGGCTCCTCGCACTCCGGCTCCTGCCAG \\
\hline$\beta 4$ E1493R Forward & CAGGGCTACAGTGTGCGGTACCAGCTGCTG \\
\hline$\beta 4$ E1493R Reverse & CAGCAGCTGGTACCGCACACTGTAGCCCTG \\
\hline$\beta 4$ N1498R Forward & GTACCAGCTGCTGCGCGGCGGTGAGCTGC \\
\hline$\beta 4$ N1498R Reverse & GCAGCTCACCGCCGCGCAGCAGCTGGTAC \\
\hline$\beta 4$ E1501R Forward & СTGCTGAACGGCGGTCGGCTGCATCGGCTC \\
\hline$\beta 4$ E1501R Reverse & GAGCCGATGCAGCCGACCGCCGTTCAGCAG \\
\hline$\beta 4$ R1504E Forward & GCGGTGAGCTGCATGAGCTCAACATCCCCAAC \\
\hline$\beta 4$ R1504E Reverse & GTTGGGGATGTTGAGCTCATGCAGCTCACCGC \\
\hline$\beta 4$ Q1512R Forward & CCCAACCCTGCCCGGACCTCGGTGGTGG \\
\hline$\beta 4$ Q1512R Reverse & CCACCACCGAGGTCCGGGCAGGGTTGGG \\
\hline$\beta 4$ Q1535R Forward & GGCCCAGAGCCGGGAAGGCTGGGGC \\
\hline$\beta 4$ Q1535R Reverse & GCCCCAGCCTTCCCGGCTCTGGGCC \\
\hline$\beta 4$ Q1535A Forward & CGGGCCCAGAGCGCGGAAGGCTGGGG \\
\hline$\beta 4$ Q1535A Reverse & СCCCAGCCTTCCGCGCTCTGGGCCCG \\
\hline$\beta 4$ Q1536R Forward & GGCCCAGAGCCAGCGAGGCTGGGGC \\
\hline$\beta 4$ Q1536R Reverse & GCCCCAGCCTCGCTGGCTCTGGGCC \\
\hline$\beta 4$ Q1536A Forward & GCCCAGAGCCAGGCAGGCTGGGGCCG \\
\hline$\beta 4$ Q1536A Reverse & CGGCCCCAGCCTGCCTGGCTCTGGGC \\
\hline$\beta 4$ R1540E-R1542E Forward & GGCTGGGGCGAAGAGGAAGAGGGTGTCATCACC \\
\hline$\beta 4$ R1540E-R1542E Reverse & GGTGATGACACCСTCTTCCTCTTCGCCCCAGCC \\
\hline
\end{tabular}


Table S6. Primers used for site directed mutagenesis of $\beta 4$ (continuation), Related to STAR Methods.

\begin{tabular}{|c|c|}
\hline Primer name & Sequence (5' - 3') \\
\hline$\beta 4$ R1540E Forward & GGAAGGCTGGGGCGAAGAGCGTGAGGGTG \\
\hline$\beta 4$ R1540E Reverse & САСССТCACGCTCTTCGCCCCAGCCTTCC \\
\hline$\beta 4$ R1540A Forward & GGAAGGCTGGGGCGCAGAGCGTGAGGGTG \\
\hline$\beta 4$ R1540A Reverse & САСССТCACGCTCTGCGCCCCAGCCTTCC \\
\hline$\beta 4$ R1542E Forward & GCTGGGGCCGAGAGGAAGAGGGTGTCATCACC \\
\hline$\beta 4$ R1542E Reverse & GGTGATGACACCCTCTTCCTCTCGGCCCCAGC \\
\hline$\beta 4$ R1542A Forward & GCTGGGGCCGAGAGGCTGAGGGTGTCATC \\
\hline$\beta 4$ R1542A Reverse & GATGACACCCTCAGCCTCTCGGCCCCAGC \\
\hline$\beta 4$ E1541R-E1543R Forward & GGCTGGGGCCGACGGCGTCGGGGTGTCATCACC \\
\hline$\beta 4$ E1541R-E1543R Reverse & GGTGATGACACCCCGACGCCGTCGGCCCCAGCC \\
\hline$\beta 4$ T1547R Forward & GGGTGTCATCCGCATTGAATCCCAGGTG \\
\hline$\beta 4$ T1547R Reverse & CACCTGGGATTCAATGCGGATGACACCC \\
\hline$\beta 4$ P1576R Forward & CAGTGCCCCAGGCCGGCTGGTGTTCACTGC \\
\hline$\beta 4$ P1576R Reverse & GCAGTGAACACCAGCCGGCCTGGGGCACTG \\
\hline$\beta 4$ V1578R-T1580R Forward & CCAGGCCCGCTGCGGTTCCGTGCCCTGAGCCC \\
\hline$\beta 4$ V1578R-T1580R Reverse & GGGCTCAGGGCACGGAACCGCAGCGGGCCTGG \\
\hline$\beta 4$ R1595E-R1596E Forward & GCTGGGAGCGGCCAGAGGAGCCCAATGGGG \\
\hline$\beta 4$ R1595E-R1596E Reverse & CCCCATTGGGCTCCTCTGGCCGCTCCCAGC \\
\hline$\beta 4$ D1600R Forward & GCCCAATGGGCGTATCGTCGGCTACCTGG \\
\hline$\beta 4$ D1600R Reverse & CCAGGTAGCCGACGATACGCCCATTGGGC \\
\hline$\beta 4$ P1616R-T1618R Forward & CAAGGAGGAGGGCGAGCCCGCGCATTCCGGG \\
\hline$\beta 4$ P1616R-T1618R Reverse & CCCGGAATGCGCGGGCTCGCCCTCCTCCTTG \\
\hline$\beta 4$ R1621E-D1623R Forward & GCCACCGCATTCGAGGTGCGTGGAGACAGCCCC \\
\hline$\beta 4$ R1621E-D1623R Reverse & GGGGCTGTCTCCACGCACCTCGAATGCGGTGGC \\
\hline$\beta 4$ E1628R-R1630E Forward & GGAGACAGCCCCCGGAGCGAGCTGACCGTGC \\
\hline$\beta 4$ E1628R-R1630E Reverse & GCACGGTCAGCTCGCTCCGGGGGCTGTCTCC \\
\hline$\beta 4$ T1632R-P1634R Forward & GAGAGCCGGCTGCGCGTGCGGGGCCTCAG \\
\hline$\beta 4$ T1632R-P1634R Reverse & CTGAGGCCCCGCACGCGCAGCCGGCTCTC \\
\hline$\beta 4$ S1637R-E1638R Forward & GCCGGGCCTCAGGCGGAACGTGCCCTAC \\
\hline$\beta 4$ S1637R-E1638R Reverse & GTAGGGCACGTTCCGCCTGAGGCCCGGC \\
\hline$\beta 4$ F1654R Forward & CCACTGAGGGCCGCGGGCCAGAGCG \\
\hline$\beta 4$ F1654R Reverse & CGCTCTGGCCCGCGGCCCTCAGTGG \\
\hline$\beta 4$ I1661R-T1663R Forward & GAGCGCGAGGGCCGCATCCGCATAGAGTCCC \\
\hline$\beta 4$ I1661R-T1663R Reverse & GGGACTCTATGCGGATGCGGCCCTCGCGCTC \\
\hline$\beta 4$ I1661R Forward & GAGCGCGAGGGCCGCATCACCATAGAGTC \\
\hline$\beta 4$ I1661R Reverse & GACTCTATGGTGATGCGGCCCTCGCGCTC \\
\hline
\end{tabular}


Table S6. Primers used for site directed mutagenesis of $\beta 4$ (continuation), Related to STAR Methods.

\begin{tabular}{|l|l|}
\hline Primer name & Sequence (5' - 3') \\
\hline$\beta 4$ T1663R Forward & CGAGGGCATCATCCGCATAGAGTCCCAGGATG \\
\hline$\beta 4$ T1663R Reverse & CATCCTGGACTCTATGCGGATGATGCCCTCG \\
\hline$\beta 4$ T1663R Forward (with Stop codon) & GCGAGGGCATCATCCGCATAGAGTCCTAGGG \\
\hline$\beta 4$ T1663R Reverse (with Stop codon) & CCCTAGGACTCTATGCGGATGATGCCCTCGC \\
\hline$\beta 4$ T1663D Forward (with Stop codon) & GCGAGGGCATCATCGACATAGAGTCCTAGGG \\
\hline$\beta 4$ T1663D Reverse (with Stop codon) & CCCTAGGACTCTATGTCGATGATGCCCTCGC \\
\hline$\beta 4$ C1483S Forward & GGCAGGAGCCGCGGTCCGAGCGGCCGCTGC \\
\hline$\beta 4$ C1483S Reverse & GCAGCGGCCGCTCGGACCGCGGCTCCTGCC \\
\hline$\beta 4$ C1483S-R1485C Forward & GGAGCCGCGGTCCGAGTGCCCGCTGCAGGGC \\
\hline$\beta 4$ C1483S-R1485C Reverse & GCCCTGCAGCGGGCACTCGGACCGCGGCTCC \\
\hline$\beta 4$ L1497C Forward & AGTGTGGAGTACCAGCTGTGTAACGGCGGTGAGCTGCATCG \\
\hline$\beta 4$ L1497C Reverse & GATGCAGCTCACCGCCGTTACACAGCTGGTACTCCACACTG \\
\hline$\beta 4$ R1504C Forward & GGCGGTGAGCTGCATTGCCTCAACATCCCCAAC \\
\hline$\beta 4$ R1504C Reverse & GTTGGGGATGTTGAGGCAATGCAGCTCACCGCC \\
\hline$\beta 4$ C1559A Forward & GCAGAGCCCACTGGCTCCCCTGCCAGGCTC \\
\hline$\beta 4$ C1559A Reverse & GCCTGGCAGGGGAGCAGTGGGCTCTGCGG \\
\hline
\end{tabular}

Table S7. Primers used for site directed mutagenesis of BP230, Related to STAR Methods.

\begin{tabular}{|l|l|}
\hline Primer name & Sequence (5' - 3') \\
\hline BP230 S27D Forward & GCACTCGAACAGTCTTGATGATAATGAAAATCTTCTCTTGG \\
\hline BP230 S27D Reverse & CCAAGAGAAGATTTTCATTATCATCAAGACTGGTTCGAGTGC \\
\hline BP230 T39D Forward & GGTTCATTGTGGTCCAGATCTGATCAACTCTTGCATTAG \\
\hline BP230 T39D Reverse & GCAAGAGTTGATCAGATCTGGACCACAATGAACCAAGA \\
\hline BP230 S43D Forward & GTCCAACACTGATCAACGATTGCATTAGCTTCGGC \\
\hline BP230 S43D Reverse & GCCGAAGCTAATGCAATCGTTGATCAGTGTTGGAC \\
\hline BP230 S46D Forward & CTGATCAACTCTTGCATTGACTTCGGCAGTGAATCC \\
\hline BP230 S46D Reverse & GGATTCACTGCCGAAGTCAATGCAAGAGTTGATCAG \\
\hline BP230 S46A Forward & CTGATCAACTCTTGCATTGCCTTCGGCAGTGAATCC \\
\hline BP230 S46A Reverse & GGATTCACTGCCGAGGCAATGCAAGAGTTGATCAG \\
\hline BP230 S49D Forward & CTTGCATTAGCTTCGGCGATGAATCCTTTGATGGAC \\
\hline BP230 S49D Reverse & GTCCATCAAAGGATTCATCGCCGAAGCTAATGCAAG \\
\hline BP230 S51D Forward & AGCTTCGGCAGTGAAGACTTTGATGGACACAGG \\
\hline BP230 S51D Reverse & ACCTGTGTCCATCAAAGTCTTCACTGCCGAAGC \\
\hline
\end{tabular}

